\title{
PRESCRIBING CURVATURE ON COMPACT SURFACES WITH CONICAL SINGULARITIES
}

\author{
MARC TROYANOV
}

\begin{abstract}
We study the Berger-Nirenberg problem on surfaces with conical singularities, i.e. we discuss conditions under which a function on a Riemann surface is the Gaussian curvature of some conformal metric with a prescribed set of singularities of conical types.
\end{abstract}

\section{INTRODUCTION}

During the last two decades, a lot of work has been done to understand which real functions defined on a surface $S$ equipped with a conformal structure are curvature of some pointwise conformal metric $d s^{2}$ on $S$. For a survey of this theory, the reader can consult [1, Chapter 5] or [9].

The first case that has been handled is that of compact surfaces with negative curvature. It is the nicest and simplest result one could hope for that has been obtained:

Theorem (Melvyn Berger [2]). On a compact Riemann surface of genus at least two, any smooth negative function is the curvature of a unique conformal metric.

In this paper, we obtain criteria for a function $S$, defined on a surface with conical singularities and/or corners, to be the curvature of a Riemannian metric compatible with a given conformal structure. Our first result parallels Berger's theorem:

Theorem A. Let $S$ be a compact Riemann surface. Let $p_{1}, p_{2}, \ldots, p_{n}$ be points of $S$ and $\theta_{1}, \theta_{2}, \ldots, \theta_{n}$ be positive numbers. Assume

$$
2 \pi \chi(S)+\sum_{i=1}^{n}\left(\theta_{i}-2 \pi\right)<0 .
$$

Then any smooth negative function on $S$ is the curvature of a unique conformal metric having at $p_{i}$ a conical singularity of angle $\theta_{i}$.

(Conical singularities are defined below.)

Received by the editors April 4, 1989.

1980 Mathematics Subject Classification (1985 Revision). Primary 58G30, 53C20.

Key words and phrases. Surfaces, curvature, conical singularities. 
Not long after Berger's theorem, Kazdan and Warner proved a theorem providing necessary and sufficient conditions for a function to be the curvature of a conformal metric on a torus:

Theorem [10]. Let $S$ be a Riemann surface of genus 1. Then a smooth function $K: S \rightarrow \mathbf{R}(\not \equiv 0)$ is the curvature of a conformal metric on $S$ if and only if

(i) $K$ changes sign,

(ii) $\int_{S} K d A<0$, where $d A$ is the area element of a conformal flat metric on $S$.

In a previous work, we obtained the following proposition (of which an alternative proof is given in the present paper):

Proposition [17]. Let $S$ be a compact Riemann surface. Let $p_{1}, p_{2}, \ldots, p_{n}$ be points of $S$ and $\theta_{1}, \theta_{2}, \ldots, \theta_{n}$ be positive numbers. Assume

$$
2 \pi \chi(S)+\sum_{i=1}^{n}\left(\theta_{i}-2 \pi\right)=0 .
$$

Then there exists a conformal flat metric on $S$ with conical singularity of angle $\theta_{i}$ at $p_{i}(\forall i)$. This metric is unique up to homothety.

Our next result is similar to Kazdan and Warner's theorem:

Theorem B. Let $S$ be a compact Riemann surface. Let $p_{1}, p_{2}, \ldots, p_{n}$ be points of $S$ and $\theta_{1}, \theta_{2}, \ldots, \theta_{n}$ be positive numbers. Assume

$$
2 \pi \chi(S)+\sum_{i=1}^{n}\left(\theta_{i}-2 \pi\right)=0 .
$$

Then a smooth function $K: S \rightarrow \mathbf{R}(\not \equiv 0)$ is the curvature of a conformal metric on $S$ with conical singularities of angle $\theta_{i}$ at $p_{i}$ if and only if

(i) $K$ changes sign,

(ii) $\int_{S} K d A<0$,

where $d A$ is the area element of a conformal flat metric (having the desired singularities) on $S$.

Unfortunately, life is not so simple in the case of the sphere, and, for instance, Kazdan and Warner [10] and Bourguignon and Ezin [3] haved constructed functions that are curvature of no conformal metric on the sphere (with its usual conformal structure).

But, on the other hand, the case of the projective plane is completely understood thanks to Moser's work (the projective plane is not a Riemann surface, but the theory works also in the nonorientable case).

Theorem [13]. Let $K: \mathbf{R} \mathbf{P}^{2} \rightarrow \mathbf{R}$ be a smooth function positive at some point. Then there exists a metric on $\mathbf{R P}^{2}$, compatible with its canonical conformal structure, and having $K$ as curvature.

What is crucial in the proof of this theorem is $0<\chi\left(\mathbf{R P} \mathbf{P}^{2}\right)<2$.

Our last result generalizes Moser's theorem: 
Theorem C. Let $S$ be a compact Riemann surface. Let $p_{1}, p_{2}, \ldots, p_{n}$ be points of $S$ and $\theta_{1} \leq \theta_{2} \leq \cdots \leq \theta_{n}$ be positive numbers. Assume

$$
0<2 \pi \chi(S)+\sum_{i=1}^{n}\left(\theta_{i}-2 \pi\right)<\min \left\{4 \pi, 2 \theta_{1}\right\} .
$$

Then any smooth function on $S$, which is positive at some point is the curvature of a conformal metric having at $p_{i}$ a conical singularity of angle $\theta_{i}$.

Our results extend to nonorientable surfaces and to surfaces with (piecewise geodesic) boundary. (Let us mention that some results in the presence of geodesic curvature for the boundary, in the smooth case, have been obtained by Cherrier [5].)

Let us stress that our discussion here (and in the rest of the paper), concerns pointwise conformal metric. For "simply" conformal metric, the results are much simpler (the only obstruction comes from the Gauss-Bonnet formula, see [11, Theorem 5.1]). Recall that a metric on a Riemann surface is pointwise conformal if it locally reads $d s^{2}=\rho(x, y)\left(d x^{2}+d y^{2}\right)$ where $z=x+i y$ is a complex parameter on the surface, and a metric is simply conformal if it is the pullback by a diffeomorphism of a pointwise conformal metric.

The paper is organized as follows:

$\S 1$ Generalized Riemann surfaces with divisors;

$\S 2$ A Gauss-Bonnet formula;

$\S 3$ Statements of the results, examples;

$\S 4$ Analysis on a surface with conical singularities;

$\S 5$ Study of the equation $\Delta u=h e^{2 u}-h_{0}$, proof of the main theorems; Appendix: A weighted Sobolev inequality.

In the first two sections, we develop general considerations about Riemannian surfaces with conical singularities. In the third section, we present our results and examples. In the last two sections, we study the techniques used to prove the theorems. The actual proof is given in $\S 5.8$.

Let us now say a word about our methods. Suppose we are given a surface $S$ with a conformal structure, and a function $K: S \rightarrow \mathbf{R}$. The question is

Is there a conformal metric on $S$ with curvature $K$ ?

To investigate this question, we use the same technique as in [2], i.e., we choose some conformal metric $d s_{0}^{2}$ (sometimes called the base metric) on $S$ and try to "conformally deform" this metric: $d s^{2}=e^{2 u} d s_{0}^{2}$ (where $u: S \rightarrow \mathbf{R}$ is some function). We know (see top of p. 16 in [10]) that a metric of this type will have curvature $K$ if and only if $u$ is a solution of the nonlinear elliptic partial differential equation

$$
\Delta u=K e^{2 u}-K_{0},
$$

where $K_{0}$ is the curvature of $d s_{0}^{2}$. (Our convention for the Laplacian is such that if $d s^{2}=\rho(x, y)\left(d x^{2}+d y^{2}\right)$, then $\Delta=-1 / \rho\left(\partial^{2} / \partial x^{2}+\partial^{2} / \partial y^{2}\right)$.) 
Thus, to answer $(*)$, we have to study the above equation. To this aim, we use the variational method.

Let us now define our singularities. By definition, a point $p \in S$ is a conical singularity of order $\beta$ (or of angle $\theta:=2 \pi(\beta+1)$ ) of the metric $d s^{2}$ if there exists a nonsingular conformal map $z: U \rightarrow \mathbf{C}$ defined in a neighbourhoood $U$ of $p$ such that $z(p)=0$ and $d s^{2}=\rho(z)|z|^{2 \beta}|d z|^{2}$ in $U$, for some continuous positive function $\rho$.

The information considering the singularities is coded in a divisor: a metric having conical singularities of order $\beta_{1}, \beta_{2}, \ldots, \beta_{n}$ at $p_{1}, p_{2}, \ldots, p_{n} \in S$ is said to represent the divisor $\boldsymbol{\beta}:=\sum_{i=1}^{n} \beta_{i} p_{i}$.

To study $(*)$ for metrics with conical singularities, we simply start with a base metric having the desired singularities, and consider only continuous conformal deformations (so that $d s_{0}^{2}$ and $d s^{2}=e^{2 u} d s_{0}^{2}$ both represent the same divisor). Thus, to answer $(*)$, we have to study a nonlinear partial differential equation on the singular Riemannian surface $\left(S, d s_{0}^{2}\right)$.

Notice that, in the first decade of this century, Emile Picard-probably motivated by the uniformisation problem for Riemann surfaces with branch pointsalready studied the above equation with $K=-1$ and conical singularities. His results correspond to our Theorem A in the case $K \equiv-1$ and $S=S^{2}$, see [16].

Independently from our work, Robert McOwen [15] also obtained our Theorem A for $K \equiv-1$ (on any compact surface).

The results of this paper are part of my thesis at Geneva University [18]. I am happy to have the opportunity to thank Professor André Haefliger, my advisor, for his constant support throughout this work. It is my pleasure to thank Henri Maire for his guidance when I had to face subtle analytical questions, and Professors J. Moser and J. P. Bourguignon for their interest in my work and for the stimulating discussions I had with them.

\section{Generalized Riemann SURFACES With Divisors}

1.1 Definitions. A generalized Riemann surface (G.R.S.) is a topological surface (perhaps with boundary) $S$ with an atlas $\left\{\phi_{i}: U_{i} \rightarrow \mathscr{H}\right\}$ where $\mathscr{H}=\{z \in$ C: $\operatorname{Im}(z) \geq 0\}$, such that the coordinate changes $\phi_{i} \circ \phi_{j}^{-1}$ are, wherever defined, conformal (i.e., holomorphic or antiholomorphic) mappings. As usual, two such atlases will be considered to define the same structure on $S$ if their union is still such an atlas.

An oriented generalized Riemann surface without boundary obviously has a natural structure of Riemann surface.

A conformal (singular) Riemannian metric on a G.R.S. $S$ is defined by a local expression

$$
d s^{2}=\rho_{i}\left(z_{i}\right)\left|d z_{i}\right|^{2},
$$

where $z_{i}$ is a local coordinate on $S$ and $\rho_{i}$ is a positive measurable function. 
A (real) divisor on a G.R.S. $S$ is simply a formal sum

$$
\boldsymbol{\beta}=\sum_{i} \beta_{i} p_{i},
$$

where the $p_{i}$ are points of $S$ and the $\beta_{i}$ are real numbers. The set $\left\{p_{i}\right\}$ (which is assumed to be discrete) is the support of $\boldsymbol{\beta}$, denoted by $\operatorname{supp}(\boldsymbol{\beta})$; the number $|\boldsymbol{\beta}|:=\sum_{i} \beta_{i}$ is the degree of the divisor.

We shall always assume that a divisor $\beta=\sum_{i} \beta_{i} p_{i}$ satisfies the following condition:

$$
\beta_{i}>-1 \text { if } p_{i} \notin \partial S \text { and } \beta_{i}>-\frac{1}{2} \text { if } p_{i} \in \partial S .
$$

Let $S$ be a G.R.S., $\boldsymbol{\beta}=\sum_{i} \beta_{i} p_{i}$ be a divisor satisfying (1.2). A conformal metric $d s^{2}$ on $S$ is said to represent the divisor $\boldsymbol{\beta}$ if $d s^{2}$ is a smooth (i.e., of class $C^{2}$ ) Riemannian metric on $S \backslash \operatorname{supp}(\boldsymbol{\beta})$ such that if $z_{i}$ is a coordinate defined in a neighbourhood $U_{i}$ of $p_{i}$, then there exists a continuous function $u: U_{i} \rightarrow \mathbf{R}$, which is of class $C^{2}$ on $U_{i}-\left\{p_{i}\right\}$, and such that in $U_{i}$ :

$$
\begin{cases}d s^{2}=e^{2 u}\left|z_{i}-a_{i}\right|^{2 \beta_{i}}\left|d z_{i}\right|^{2} & \text { if } p_{i} \notin \partial S, \\ d s^{2}=e^{2 u}\left|z_{i}-a_{i}\right|^{4 \beta_{i}}\left|d z_{i}\right|^{2} & \text { if } p_{i} \in \partial S,\end{cases}
$$

where $a_{i}=z_{i}\left(p_{i}\right)$.

The point $p_{i}$ is then said to be a conical singularity of angle $\theta_{i}=2 \pi\left(\beta_{i}+1\right)$ if $p_{i} \notin \partial S$, and a corner of angle $\varphi_{i}=2 \pi\left(\beta_{i}+\frac{1}{2}\right)$ (or of "exterior angle" $\left.-2 \pi \beta_{i}\right)$ if $p_{i} \in \partial S$. In both cases, we will simply say that $d s^{2}$ has a singularity of $\operatorname{order} \beta_{i}$ at $p_{i}$.

Observe that $\mathbf{C}$, equipped with the metric $|z|^{2 \beta}|d z|^{2}$, is isometric to an euclidean cone of total angle $\theta=2 \pi(\beta+1)$. Thus, if $d s^{2}$ has a conical singularity of order $\beta$ at $p$, then $d s^{2}$ admits a "tangent cone" of angle $\theta$ at this point. In other words, the number $\beta_{i}$ measures the "number of turns" in excess at the point $p_{i}$.

1.2 Examples. (1) Let $S$ be a Riemann surface and $\omega$ be a holomorphic differential on $S$, then $d s^{2}:=|\omega|^{2}$ is a (flat) conformal metric on $S$ representing the divisor $\boldsymbol{\beta}=\operatorname{div}(\omega)$.

(2) Let $\left(S_{1}, d s_{1}^{2}\right)$ be a smooth Riemannian surface, and $f: S \rightarrow S_{1}$ be a branched covering, then $d s^{2}:=f^{*}\left(d s_{1}^{2}\right)$ is a metric on $S$ representing the "ramification divisor" $\boldsymbol{\beta}=\sum O_{p}(f) p$, (where $O_{p}(f)$ is the ramification order of $f$ at the point $p \in S$ ).

(3) If $S$ is a surface with a polyhedral metric, then this metric is Riemannian and represents the divisor $\boldsymbol{\beta}=\sum \beta_{i} p_{i}$, where $p_{i}$ ranges through the set of vertices, and $\beta_{i}=\theta_{i} / 2 \pi-1$ if $p_{i}$ is an interior point of total angle $\theta_{i}$, and $\beta_{i}=\varphi_{i} / 2 \pi-\frac{1}{2}$ if $p_{i}$ is a boundary point of angle $\varphi_{i}$.

(4) If $\left(\widetilde{S}, d \tilde{s}^{2}\right)$ is a smooth Riemannian surface on which a finite group $G$ acts by isometries, then $S=\widetilde{S} / G$ carries a metric $d s^{2}$ representing the divisor 
$\boldsymbol{\beta}=\sum \beta_{p} p$ where $\beta_{p}$ is determined by the action of $G$ on $\widetilde{S}$ in the following way. If $\tilde{p} \in \widetilde{S}$ represents $p \in S$, we note $I(\tilde{p}) \subset G$ the isotropy group of $\tilde{p}$. Then, $\beta_{p}:=(1 / n)-1$ if $I(\tilde{p})$ is a rotation group of order $n, \beta_{p}=(1 / 2 k)-\frac{1}{2}$ if $I(\tilde{p})$ is a dihedral group of order $2 k$, and $\beta_{p}=-\frac{1}{4}$ if $\tilde{p}$ is a boundary point and $I(\tilde{p})$ is a symmetry group of order 2 .

1.3 Conformal mapping. There is a natural class of mappings between two G.R.S.'s. These are the conformal mappings. From a topological viewpoint, these mappings are covering maps in the sense of orbifold theory (in dimension 2).

A map $f: S^{\prime} \rightarrow S$ between two G.R.S.'s is said to be conformal if it is continuous and, whenever $z$ is a coordinate defined in an open set $U \subset S^{\prime}$, and $w$ a coordinate defined in $V \subset S$ ( $U$ being such that $f(U) \subset V)$, then $w:=f(z)$ defines a nonconstant holomorphic or antiholomorphic map.

A point $p^{\prime} \in S^{\prime}$ is a regular point of $f$ if $f$ is one-to-one in a neighbourhood of $p^{\prime}$, it is a singular point of $f$ otherwise.

Lemma 1. Let $p^{\prime} \in S^{\prime}$ be a singular point of a conformal map $f: S^{\prime} \rightarrow S$, and denote by $p=f\left(p^{\prime}\right)$ its image. Then the singularity has one of the following types:

Type I: $(p \notin \partial S)$. There exist coordinates $z$ in a neighbourhood of $p^{\prime}, w$ in neighbourhood of $p$ and an integer $m$ such that

$$
w=f(z)=a+\left(z-a^{\prime}\right)^{m+1} .
$$

Type II: $\left(p^{\prime} \in \partial S^{\prime}\right)$. There exist coordinates $z$ in a neighbourhood of $p^{\prime}, w$ in a neighbourhood of $p$ and an integer $m$ such that

$$
w=f(z)=a+\Phi\left(\left(z-a^{\prime}\right)^{m+1}\right) .
$$

Type III: $\left(p^{\prime} \notin \partial S^{\prime}, p \in \partial S\right)$. There exist coordinates $z$ in a neighbourhood of $p^{\prime}, w$ in a neighbourhood of $p$ and an odd integer $m$ such that

$$
w=f(z)=a+\Phi\left(\left(z-a^{\prime}\right)^{(m+1) / 2}\right) .
$$

Where $a=w(p), a^{\prime}=z\left(p^{\prime}\right)$, and $\Phi$ is the map $\mathbf{C} \rightarrow \mathbf{C}$ defined by $\Phi(x+i y)=x+i|y|$.

The integer $m$ is called the branching order of $f$ at $p^{\prime}$. Singularities of Type III are called folds if the branching order $m$ equals 1 .

Proof. This is an immediate consequence of the classificaion of singularities of holomorphic functions.

Observe that, off the singularities, the map $f$ is a covering map and hence, the number of preimages of all regular points is constant (assuming $S^{\prime}$ connected). This number is the number of leaves or the degree of $f$.

Examples. (1) If $S^{\prime}$ and $S$ are Riemann surfaces and $f$ is a holomorphic map, then it is a conformal map, and the only possible singularities are of Type I. 
(2) If $S^{\prime}=\left\{(x, y, z) \in \mathbf{R}^{3}: x^{2}+y^{2}+z^{2}=1\right\}$ is the usual sphere, $S=$ $\left\{(x, y, z) \in S^{\prime}: z \geq 0\right\}$ the upper hemisphere, and $f: S^{\prime} \rightarrow S$ is defined by $f(x, y, z)=(x, y,|z|)$, then $f$ is conformal of degree 2 and the singularities are folds lying on the equator $\{z=0\}$.

(3) The map $f: \mathbf{C} \rightarrow S:=\{x+i y: x \geq 0, y \geq 0\}$ defined by $f(x+i y)=$ $|x|+i|y|$ is conformal. It has folds on the axis (off the origin), and a singularity of Type III and branching order $m=3$ at 0 . (Indeed, if one considers the coordinate $w=(x+i y)^{2}$ on $S$, then $\left.w=f(z)=\Phi\left(z^{2}\right)\right)$.

(4) More generally, if $\left(S^{\prime}, d s^{\prime 2}\right)$ is a Riemannian surface (perhaps with conical singularities and corners) admitting a discrete group $G$ of isometries, then the canonical projection $f: S^{\prime} \rightarrow S:=S^{\prime} / G$ is a conformal mapping with singularities at points of nontrivial isotropy. Its degree is the order of $G$.

Suppose we are given a conformal mapping $f: S^{\prime} \rightarrow S$ between two G.R.S.'s and a real divisor $\boldsymbol{\beta}=\sum \beta_{p} p$ on $S$. Then, we define a divisor $\boldsymbol{\beta}^{\prime}$ (also denoted by $\left.f^{*} \boldsymbol{\beta}\right)$ on $S^{\prime}$ by $\boldsymbol{\beta}^{\prime}=\sum \beta_{p^{\prime}}^{\prime} p^{\prime}$ where

$$
\beta_{p^{\prime}}^{\prime}= \begin{cases}\beta_{f\left(p^{\prime}\right)} & \text { if } p^{\prime} \text { is regular; } \\ m+(m+1) \beta_{f\left(p^{\prime}\right)} & \text { if } p^{\prime} \text { is of Type I; } \\ m / 2+(m+1) \beta_{f\left(p^{\prime}\right)} & \text { if } p^{\prime} \text { is of Type II; } \\ (m-1) / 2+(m+1) \beta_{f\left(p^{\prime}\right)} & \text { if } p^{\prime} \text { is of Type III. }\end{cases}
$$

Where $m$ is the branching order of $p^{\prime}$.

If $\boldsymbol{\beta}=0$, then $\boldsymbol{\beta}^{\prime}$ is not zero, unless $f$ has no singularities, and it is called the "ramification divisor of $f$ ". Its degree $\left|\boldsymbol{\beta}^{\prime}\right|$ is the "total branching order" of $f$.

1.4 The canonical double cover of a compact G.R.S. The next proposition will say that a compact G.R.S. can be thought of as a closed Riemann surface modulo an involution. Let us first give a few examples:

(1) If $S$ is orientable and $\partial S \neq \varnothing$, then $S=S^{\prime} / \sigma$ where $S^{\prime}$ is the "double" of $S$ (i.e., two copies of $S$ glued together along their boundary), and the involution $\sigma$ exchanges the two copies of $S$.

(2) If $S$ is nonorientable and $\partial S=\varnothing$, then $S^{\prime}$ is the classical "orientation cover" of $S$.

(3) Let $S$ be the surface obtained from the triangle $\{(x, y): 0 \leq x \leq$ $y \leq 1\}$ by identifying $(x, 1)$ with $(0, x)$ (hence $S$ is homeomorphic to a Möbius band). Then $S^{\prime}$ is the torus $\{(x, y) \bmod \mathbf{Z}\}$, and $\sigma$ is the involution $\sigma(x, y)=(y, x)$.

Proposition 2. Let $S$ be a compact G.R.S. Then, there exists a closed Riemann surface $S^{\prime}$ and a conformal map $f: S^{\prime} \rightarrow S$ of degree 2. Furthermore, $S^{\prime}$ admits a conformal involution $\sigma: S^{\prime} \rightarrow S^{\prime}$ such that $f(p)=f(q)$ if and only if $p=q$ or $p=\sigma(q)$. Finally, the only singularities of $f$ are folds forming the fix point set of $\sigma$ (which may be empty). 
Proof. Let $\mathscr{L}$ denote the set of germs of locally one-to-one conformal maps, $f: U \rightarrow \mathscr{H}$ where $\mathscr{H}=\{z \in \mathbf{C}: \operatorname{Im} z \geq 0\}$ and $U$ is some open set in $S$. Let us denote such a germ at a point $x \in S$ by $(x, \phi)$. We define the "source" map $\hat{f}: \mathscr{L} \rightarrow S$ by $\hat{f}(x, \phi)=x$.

If we topologize $\mathscr{L}$ by the topology of germs, then $\hat{f}$ is a local homeomorphism.

We shall say that $(x, \phi)$ and $(y, \psi) \in \mathscr{L}$ are equivalent if one of the following conditions holds:

(1) $x=y \in \partial S$, or

(2) $x=y \notin \partial S$, and $\psi \circ \phi^{-1}$ is holomorphic at $x$.

We note $S^{\prime}:=\mathscr{L} / \sim$ and $f: S^{\prime} \rightarrow S$ the map induced by $\hat{f}$ on $S^{\prime}$. It is clear that $S^{\prime}$ is an orientable surface with a natural structure of Riemann surface on $f^{-1}(S \backslash \partial S)$. Thus it only remains to describe a chart in a neighbourhood of a point $x^{\prime} \in f^{-1}(\partial S)$. To this aim, choose a conformal map $\phi: U \rightarrow \mathscr{H}$ representing a germ $(x, \phi) \in \mathscr{L}$ at $x=f\left(x^{\prime}\right) \in \partial S$.

The set $\widehat{U}:=\{(y, \phi): y \in U\} \cup\{(y,-\bar{\phi}): y \in U\}$ (where $\bar{\phi}$ is the complex conjugate of $\phi)$ is open in $\mathscr{L}$. The image $U^{\prime}$ of $\widehat{U}$ in $S^{\prime}$ is thus a neighbourhood of $x^{\prime}$.

The map $\phi^{\prime}: U^{\prime} \rightarrow \mathbf{C}$, defined by $\phi^{\prime}(y, \phi)=\phi(y), \phi^{\prime}(y,-\bar{\phi})=\bar{\phi}(y)$, is the desired chart.

On $\mathscr{L}$, an involution is defined by $\sigma(x, \phi):=(x,-\bar{\phi})$. This involution is compatible with the equivalence relation $\sim$. We thus have an involution $\sigma: S^{\prime} \rightarrow S^{\prime}$ which clearly has the desired property.

\section{A GaUss-Bonnet formula}

Let $(S, \boldsymbol{\beta})$ be a compact G.R.S. with divisor $\boldsymbol{\beta}=\sum_{i} \beta_{i} p_{i}$ such that (1.2) holds. A conformal Riemannian metric $d s^{2}$ on $S$ representing $\boldsymbol{\beta}$ has a curvature function defined on the complement of the support of $\boldsymbol{\beta}$. We will make from now on the following assumption:

The curvature extends on $S$ as a Hölder-continuous function.

(A function on $S$ is Hölder-continuous if, in any local coordinate, it satisfies a Hölder condition.) If $S$ has a boundary, then the geodesic curvature is a well defined continuous function $k: \partial S \backslash \operatorname{supp}(\boldsymbol{\beta}) \rightarrow \mathbf{R}$. We also assume that:

The geodesic curvature extends continuously on $\partial S$.

If $S$ is compact, then the Euler characteristic of $(S, \boldsymbol{\beta})$ is defined to be

$$
\chi(S, \boldsymbol{\beta}):=\chi(S)+|\boldsymbol{\beta}|,
$$

where $\chi(S)$ is the topological Euler characteristic of $S$, and $|\boldsymbol{\beta}|\left(=\sum_{i} \beta_{i}\right)$ is the degree of $\boldsymbol{\beta}$. 
Proposition 1 (Gauss-Bonnet formula). Let $(S, \boldsymbol{\beta})$ be a compact G.R.S. with a divisor as above. Let $d s^{2}$ be a conformal metric representing $\boldsymbol{\beta}$. Then

$$
\frac{1}{2 \pi} \iint_{S} K d A+\frac{1}{2 \pi} \int_{\partial S} k d s=\chi(S, \boldsymbol{\beta}),
$$

where $K$ is the curvature, $d A$ the area element, and $k$ the geodesic curvature of $d s^{2}$.

Example. Let $S$ be the surface of a cube with vertices $p_{1}, p_{2}, \ldots, p_{8}$. Then its natural metric represents the divisor $\boldsymbol{\beta}=\sum_{i=1}^{8}\left(-\frac{1}{4}\right) p_{i}$. The Euler characteristic of $S$ is thus $\chi(S, \boldsymbol{\beta})=2+8 \times\left(-\frac{1}{4}\right)=0$. This is coherent with the GaussBonnet formula since the metric is flat.

Corollary 2 (Riemann-Hurwitz formula). Let $(S, \boldsymbol{\beta})$ be a compact G.R.S. with a divisor as above. If $f: S^{\prime} \rightarrow S$ is a conformal map of degree $n$, then

$$
\chi\left(S^{\prime}, \boldsymbol{\beta}^{\prime}\right)=n \chi(S, \boldsymbol{\beta}),
$$

where $\boldsymbol{\beta}^{\prime}=f^{*} \boldsymbol{\beta}$.

The proof of the corollary follows immediately from the proposition. Indeed, if $d s^{2}$ represents $\boldsymbol{\beta}$, then $f^{*} d s^{2}$ represents $\boldsymbol{\beta}^{\prime}$ (the existence of at least one conformal metric representing $\boldsymbol{\beta}$ follows easily from a partition of unity argument).

The proof of the proposition rests on the following lemma:

Lemma 3. If $d s^{2}$ has a conical singularity of order $\beta$ at $p \in S \backslash \partial S$, then

$$
|z-a| \frac{\partial u}{\partial z} \text { and }|z-a| \frac{\partial u}{\partial \bar{z}} \rightarrow 0, \quad \text { when } z \rightarrow a,
$$

where $z$ is a coordinate in a neighbourhood of $p, a=z(p)$ and $d s^{2}=$ $e^{2 u}|z-a|^{2 \beta}|d z|^{2}$.

Proof. We have

$$
\frac{\partial^{2} u}{\partial z \partial \bar{z}}=-4 K|z-a|^{2 \beta} e^{2 u}
$$

where $K$ is the curvature.

Assume first that $\beta \geq 0$. Since $u$ is (by definition) continuous as well as $K|z-a|^{2 \beta}$, the elliptic regularity implies that $u \in W_{\text {loc }}^{2, p}$ for all $p<\infty$ (see [8]). By Sobolev embedding, $u \in C^{1, \delta}$, and the lemma follows immediately. If $-1<\beta<0$, choose $m$ such that $\beta^{\prime}:=m+(m+1) \beta>0$ and set $z=a+w^{m+1}$. Lifting the metric to this branched covering, we have $d s^{2}=e^{2 u^{\prime}}|w|^{2 \beta^{\prime}}|d w|^{2}$ with $u^{\prime}=u+\log (m+1)$. Using the equation above, we see that $\partial u^{\prime} / \partial w$ is continuous, hence

$$
|z-a|\left|\frac{\partial u}{\partial z}\right|=\frac{1}{m+1}|w|\left|\frac{\partial u^{\prime}}{\partial w}\right|
$$

goes to 0 when $z \rightarrow a$. 
Proof of Proposition 1. We only give the proof in the case where $k \equiv 0$ (i.e., $\partial S$ is geodesic off the support of $\boldsymbol{\beta}$ ), since we will not use the more general case. Thanks to Proposition 2 in $\S 1.4$, it is easy to reduce the proof to the case where $S$ is without boundary and orientable. We assume therefore that $S$ is a closed Riemann surface.

Choose a smooth conformal metric $d s_{1}^{2}$ on $S$. If $K_{1}$ and $d A_{1}$ denote the curvature and the area element of $d s_{1}^{2}$, then the classical Gauss-Bonnet formula reads

$$
\frac{1}{2 \pi} \iint_{S} K_{1} d A_{1}=\chi(S) \text {. }
$$

There exists a function $v: S \rightarrow \mathbf{R}$ such that $d s^{2}=e^{2 v} d s_{1}^{2}$. We have

$$
K d A=K_{1} d A_{1}-d * d v,
$$

where $*$ is the Hodge operator on forms (i.e., $* d v$ is locally defined by $* d v=$ $-i(\partial v / \partial z) d z+i(\partial v / \partial \bar{z}) d \bar{z})$.

Thanks to equation (2.1), we only have to show that

$$
-\frac{1}{2 \pi} \iint_{S} d * d v=|\boldsymbol{\beta}| \text {. }
$$

Since $S$ is compact, $\operatorname{supp}(\boldsymbol{\beta})=\left\{p_{1}, p_{2}, \ldots, p_{n}\right\}$ is finite. Let $D_{i}(\varepsilon)$ be a disk of radius $\varepsilon$ around $p_{i}$. Choose $\varepsilon$ small enough so that all $D_{i}(\varepsilon)$ are disjoint. Let $S_{\varepsilon}:=S \backslash \bigcup_{i} D_{i}(\varepsilon)$, then by Green's theorem we have

$$
-\frac{1}{2 \pi} \iint_{S_{\varepsilon}} d * d v=\frac{1}{2 \pi} \sum_{i=1}^{n} \int_{\partial D_{i}(\varepsilon)} * d v .
$$

Fix $i \in\{1,2, \ldots, n\}$ and choose a coordinate $z$ in $D_{i}$ such that $z\left(p_{i}\right)=0$. The point $p_{i}$ being a conical singularity of order $\beta_{i}$, we have $v=\beta_{i} \log |z|+u$, where $u$ is continuous and satisfies the condition in Lemma 3. Hence

$$
\frac{1}{2 \pi} \int_{\partial D_{i}(\varepsilon)} * d v=\frac{\beta_{i}}{2 \pi} \int_{\partial D_{i}(\varepsilon)} * d \log |z|+\frac{1}{2 \pi} \int_{\partial D_{i}(\varepsilon)} * d u=\beta_{i}+\frac{1}{2 \pi} \int_{\partial D_{i}(\varepsilon)} * d u \text {. }
$$

Using Lemma 3, we have

$$
\lim _{\varepsilon \rightarrow 0} \frac{1}{2 \pi} \int_{\partial D_{i}(\varepsilon)} * d v=\beta_{i} .
$$

\section{Formulation OF THE RESUlts}

3.1. Let $(S, \boldsymbol{\beta})$ be compact G.R.S. Besides the Euler characteristic $\chi(S, \boldsymbol{\beta})$, we introduce another number: the Trudinger constant defined by

$$
\tau(S, \boldsymbol{\beta})=\left\{\begin{array}{l}
\min \{2,2+2 \alpha\} \quad \text { if } \partial S=\varnothing ; \\
\min \left\{1,1+\alpha, 1+2 \alpha^{\prime}\right\}, \quad \text { otherwise, }
\end{array}\right.
$$

where $\alpha=\min \left\{\beta_{i}: p_{i} \in S \backslash \partial S\right\}$, and $\alpha^{\prime}=\min \left\{\beta_{j}: p_{j} \in \partial S\right\}$.

Observe that if $\boldsymbol{\beta}$ satisfy (1.2), then $\tau(S, \boldsymbol{\beta})$ is positive. (Originally, this constant appeared analytically and not geometrically, see $\S 4.8$ and $\S 4.9$.) 
3.2 The negative case.

Theorem 1. Let $(S, \boldsymbol{\beta})$ be a compact connected G.R.S. with a divisor $\boldsymbol{\beta}=$ $\sum_{i} \beta_{i} p_{i}$ satisfying condition (1.2), and $K: S \rightarrow \mathbf{R}$ be a Hölder-continuous function. Assume $\chi(S, \boldsymbol{\beta})<0$ and $\sup (K)<0$, then there exists on $S$ a unique conformal metric representing $\boldsymbol{\beta}$, having $K$ as curvature and such that $\partial S \backslash \operatorname{supp}(\boldsymbol{\beta})$ is geodesic.

By Gauss-Bonnet formula, if $K$ is a negative function on $S$, then we must have $\chi(S, \boldsymbol{\beta})<0$ for $K$ to be the curvature of some metric representing $\boldsymbol{\beta}$ on $S$. The theorem says that it is a sufficient condition.

An important particular case is the following: Let $S$ be a closed Riemann surface of genus $g, p_{1}, p_{2}, \ldots, p_{n}$ be points in $S$ and $\nu_{1}, \nu_{2}, \ldots, \nu_{n} \in \mathbf{N}$ be positive integers. Assume the number

$$
A=n+2 g-2-\sum_{i=1}^{n} \frac{1}{\nu_{i}}
$$

to be positive. Then there exists a unique conformal hyperbolic metric on $S$ having at $p_{i}$ a conical singularity of order $1 / \nu_{i}-1$. Thus, $S=\mathscr{H} / \Gamma$ where $\Gamma$ is a fuchsian group with elliptic elements of order $\nu_{i}$ in the isotropy of points above $p_{i}$ (this is the uniformisation theorem for Riemann surfaces with branch points).

\subsection{The null case.}

Proposition 2. Let $(S, \boldsymbol{\beta})$ be a compact connected G.R.S. with a divisor $\boldsymbol{\beta}=$ $\sum_{i} \beta_{i} p_{i}$ satisfying condition (1.2) and such that $\chi(S, \boldsymbol{\beta})=0$.

Then there exists on $S$ a conformal flat metric representing $\boldsymbol{\beta}$, and such that $\partial S \backslash \operatorname{supp}(\boldsymbol{\beta})$ is geodesic. This metric is unique up to homothety.

This proposition has been previously obtained by a different method [16].

Theorem 3. Let $(S, \boldsymbol{\beta})$ be as in the previous proposition. Let $K: S \rightarrow \mathbf{R}$ be a Hölder-continuous function such that $K \not \equiv 0$.

Then there exists on $S$ a conformal metric representing $\boldsymbol{\beta}$, having $K$ as curvature (and such that $\partial S \backslash \operatorname{supp}(\boldsymbol{\beta})$ is geodesic) if and only if

$$
\sup (K)>0 \text { and } \iint_{S} K d A_{0}<0,
$$

where $d A_{0}$ is the area element of some conformal flat metric representing $\boldsymbol{\beta}$ on $S$.

For instance, on the surface of a cube, any function $K$ which is positive somewhere and negative in average is the curvature of some conformal metric (having the same conical singularities as the cube). 


\subsection{The positive case.}

Theorem 4. Let $(S, \boldsymbol{\beta})$ be a compact connected G.R.S. with a divisor $\boldsymbol{\beta}=$ $\sum_{i} \beta_{i} p_{i}$ satisfying the condition (1.2), and such that $0<\chi(S, \boldsymbol{\beta})<\tau(S, \boldsymbol{\beta})$.

Then a Hölder-continuous function $K: S \rightarrow \mathbf{R}$ is the curvature of some conformal metric representing $\boldsymbol{\beta}$ (and such that $\partial S \backslash \operatorname{supp}(\boldsymbol{\beta})$ is geodesic) if and only if $\sup (K)>0$.

Examples. (1) Choose a spherical triangle $T \subset S^{2}$ with vertices $p_{1}, p_{2}, p_{3}$ of angles $\frac{1}{2} \theta_{1}, \frac{1}{2} \theta_{2}, \frac{1}{2} \theta_{3}\left(\theta_{i}<2 \pi\right)$. Glueing two copies of $T$ isometrically along its boundary, one obtains a Riemannian surface $S$ (homeomorphic to a sphere) with constant positive curvature and three conical singularities $p_{1}, p_{2}, p_{3}$ of angles $\theta_{1}, \theta_{2}, \theta_{3}$. The classical conditions on the angles of a spherical triangle

$$
\pi<\frac{1}{2} \theta_{1}+\frac{1}{2} \theta_{2}+\frac{1}{2} \theta_{3}<\min \left\{\theta_{i}\right\}+\pi,
$$

are equivalent to the conditions $0<\chi(S, \boldsymbol{\beta})<\tau(S, \boldsymbol{\beta})$. Hence, any function positive somewhere on $S$ is the curvature of some conformal metric having at $p_{i}$ a conical singularity of angle $\theta_{i}$.

(2) Let $D=\{z:|z| \leq 1\}$ be the closed disk, $p_{1}, p_{2}, \ldots, p_{n}$ be $n$ points on the boundary $\partial D$ and $\varphi_{1}, \varphi_{2}, \ldots, \varphi_{n}$ be positive numbers such that

$$
(n-2) \pi<\sum_{i=1}^{n} \varphi_{i}<2 \min \left\{\varphi_{i}\right\}+(n-2) \pi .
$$

Then any function positive somewhere on $D$ is the curvature of some conformal metric having at $p_{i}$ a corner of angle $\varphi_{i}$ and such that $\partial D \backslash\left\{p_{i}\right\}$ is geodesic.

Observe that the arithmetical condition on the $\varphi_{i}$ is exactly the condition of existence of a spherical polygon with angles $\varphi_{1}, \varphi_{2}, \ldots, \varphi_{n}$.

3.5 Critical and "supercritical" surfaces with divisor. We shall say that a compact G.R.S. with divisor $(S, \boldsymbol{\beta})$ is subcritical if

$$
0<\chi(S, \boldsymbol{\beta})<\tau(S, \boldsymbol{\beta}) \text {. }
$$

It will be said to be critical if $\chi(S, \boldsymbol{\beta})=\tau(S, \boldsymbol{\beta})$, and supercritical if $\chi(S, \boldsymbol{\beta})>$ $\tau(S, \boldsymbol{\beta})$.

For instance, the sphere (with trivial divisor), the disk (with trivial divisor), the sphere with divisor $\beta p+\beta q \quad(\beta<0)$ and the disk with divisor $\beta p \quad(\beta<$ $0, p \notin \partial D)$ are critical surfaces. The sphere with divisor $\beta_{1} p_{1}+\beta_{2} p_{2}(0>$ $\left.\beta_{1} \neq \beta_{2}\right)$ and the disk with divisor $\beta p(\beta<0, p \in \partial D)$ are supercritical. The only smooth subcritical surface is the projective plane.

Theorem 4 says that on a subcritical surface, a function is the curvature of some conformal metric representing the divisor if and only if this function is positive somewhere. By contrast, there are known counterexamples on the sphere (see [10 and 3]).

In general, very little is known about the curvature of a critical or supercritical surface (we know for instance that there is no metric of constant curvature on a 
sphere with divisor $\beta_{1} p_{1}+\beta_{2} p_{2}$ if $\beta_{1} \neq \beta_{2}$ (see [19]), in particular, there is no metric with constant curvature on the sphere having a single conical singularity). However, there are some positive results, Chang and Yang give criteria for a function to be the curvature of some conformal metric on $S^{2}$ related to the structure of its critical points (see [6]).

Concerning supercritical surfaces, it should be easier to treat first the case where the metric is sufficiently flat in a neighbourhood of the singularities, for instance, we have the following result of $\mathrm{R}$. McOwen on the sphere with a single conical singularity:

Theorem 5. Let $(S, \boldsymbol{\beta})$ be the sphere $S=\mathbf{C} \cup\{\infty\}$ with the divisor $\boldsymbol{\beta}=\boldsymbol{\beta} \cdot 0$, $-1<\beta<0$. If $K$ is a Hölder-continuous function positive somewhere and such that $K(z) \leq C \cdot|z|^{l}$ for some constants $C>0, l>-\beta$, then there exists a conformal metric on $S$ representing the divisor $\boldsymbol{\beta}$.

This theorem is a consequence of Theorem 1 in [14]. (To pass from our notations to those of McOwen, set $\alpha=-(\beta+2)$ and perform the inversion $z=1 / x$.)

3.6 An application to the Nirenberg problem. The Nirenberg problem is the problem of deciding whether a given function on the standard sphere $S^{2} \subset \mathbf{R}^{3}$ is the curvature of some (pointwise) conformal metric.

Proposition 6. Let $G$ be a finite subgroup of the orthogonal group $O(3)$ such that either the orbit under the action of $G$ of any point of the sphere $S^{2} \subset \mathbf{R}^{3}$ contains at least two points and $S:=S^{2} / G$ has no boundary, or the orbit of any point of $S^{2}$ contains at least three points.

Then any smooth G-invariant function $K: S^{2} \rightarrow \mathbf{R}$ which is positive somewhere is the curvature of some conformal metric on the sphere. The metric can be chosen to be G-invariant.

Proof. Let $S$ be the orbifold $S^{2} / G$ and let $\boldsymbol{\beta}=\sum_{i=1}^{n} \beta_{i} p_{i}$ be the divisor on $S$ associated to its orbifold structure (as in Example 4 in §1.2). Assume $\beta_{1}=\min \left\{\beta_{i}\right\}$. Choose a point $q \in S^{2}$ above $p_{1} \in S$, and denote by $t$ the order of the isotry group of $q$ (i.e., $t=\#\{g \in G: g \cdot q=q\}$ ), and by $m$ the order of $G$.

Assume first that $S$ has no boundary, and observe the following simple facts:

(i) $\beta_{1} \geq \frac{1}{t}-1$;

(ii) $\tau(S, \boldsymbol{\beta})=2+2 \beta_{1}$;

(iii) $\chi(S, \boldsymbol{\beta})=\frac{2}{m}$, by Riemann-Hurwitz;

(iv) $t \leq \frac{m}{2}$, by the hypothesis on the orbits.

From which we have

$$
\tau(S, \boldsymbol{\beta})=2\left(1+\beta_{1}\right) \geq \frac{2}{t}>\frac{2}{m}=\chi(S, \boldsymbol{\beta}) .
$$

Assume next that any orbit contains at least three points (and $S$ may have a boundary). Then (i) and (iii) above still hold and we have furthermore:

(ii') $\tau(S, \boldsymbol{\beta}) \geq 1+\beta_{1}$; 
(iv') $t<\frac{m}{2}$, by the hypothesis on the orbits.

Hence

$$
\tau(S, \boldsymbol{\beta}) \geq\left(1+\beta_{1}\right) \geq \frac{1}{t}>\frac{2}{m}=\chi(S, \boldsymbol{\beta}) .
$$

Thus, in both case, $S=S^{2} / G$ is subcritical and we may apply Theorem 4 on this surface. This solves the Nirenberg problem for any $G$-invariant function on $S^{2}$.

\section{ANALYSIS ON A SURFACE WITH CONICAL SINGULARITIES}

4.1. In this section, $S$ will be a compact G.R.S. without boundary, $\boldsymbol{\beta}=$ $\sum_{i=1}^{n} \beta_{i} p_{i}$ a divisor such that $\beta_{i}>-1(\forall i)$.

We set $\alpha:=\min _{i}\left\{\beta_{i}\right\}, \omega:=\max _{i}\left\{\beta_{i}\right\}$.

We will also be given on $S$ a conformal metric $d s_{0}^{2}$, with conical singularities, representing the divisor $\boldsymbol{\beta}$, as well as a smooth conformal metric $d s_{1}^{2}$.

By definition, there exists a function $\rho: S \rightarrow \mathbf{R}$ such that

$$
d s_{0}^{2}=\rho \cdot d s_{1}^{2} .
$$

This function is smooth and positive outside of the support of $\boldsymbol{\beta}$. If $z$ is a coordinate in a neighbourhood of $p_{i}\left(\right.$ such that $\left.z\left(p_{i}\right)=0\right)$, then we have in this neighbourhood

$$
\rho(z)=O\left(|z|^{2 \beta_{i}}\right) \quad(z \rightarrow 0) .
$$

4.2. Let $d A_{i}$ denote the area element of $d s_{i}^{2}$. Then we have $d A_{0}=\rho \cdot d A_{1}$. It will be useful to compare these two measures: observe first, that it follows from (4.2) that

$$
\int_{S} \rho^{k} d A_{1}<\infty\left\{\begin{array}{l}
\text { for all } k \geq 0, \quad \text { if } \alpha \geq 0, \quad \text { or } \\
\text { for } 0 \leq k<-1 / \alpha, \quad \text { if } \alpha<0,
\end{array}\right.
$$

and

$$
\int_{S} \rho^{-k} d A_{1}<\infty\left\{\begin{array}{l}
\text { for all } k \geq 0, \quad \text { if } \omega \leq 0, \quad \text { or } \\
\text { for } 0 \leq k<1 / \omega, \quad \text { if } \omega>0 .
\end{array}\right.
$$

We will write $L^{p}\left(d s_{i}^{2}\right)$ for the Banach space of functions on $S$ which are $p$ integrable with respect to $d A_{i}$.

\section{Proposition 1.}

(i) If $p>\max \{q, q /(\alpha+1)\}$, then $L^{p}\left(d s_{1}^{2}\right) \subset L^{q}\left(d s_{0}^{2}\right)$;

(ii) If $p>\max \{q, q(\omega+1)\}$, then $L^{p}\left(d s_{0}^{2}\right) \subset L^{q}\left(d s_{1}^{2}\right)$.

Proof. (i) The first embedding is obvious if $\alpha>0$. We may thus assume $-1<\alpha<0$. Set $s:=p / q$ and $t:=p /(p-q)$, from $p>q /(\alpha+1)$, we have $\alpha t>-1$. Thus $\rho \in L^{t}\left(d s_{1}^{2}\right)$ by (4.3).

Let $f \in L^{q}\left(d s_{1}^{2}\right)$. Since $\frac{1}{t}+\frac{1}{s}=1$, we have, using Hölder's inequality,

$$
\int_{S}|f|^{q} \rho d A_{1} \leq\left(\int_{S}|f|^{s q} d A_{1}\right)^{1 / s} \cdot\left(\int_{S} \rho^{t} d A_{1}\right)^{1 / t} .
$$


Raising this inequality to the power $1 / q$ (and remembering $s q=p, \rho d A_{1}=$ $d A_{0}$ ), we get

$$
\|f\|_{L^{q}\left(d s_{0}^{2}\right)} \leq \text { const } \cdot\|f\|_{L^{p}\left(d s_{1}^{2}\right)} .
$$

(ii) The second embedding being immediate if $\omega \leq 0$, we assume $\omega>0$. Again, let $s=p / q, t=p /(p-q)$. From $p>q(\omega+1)$ follows $\omega q /(p-q)<1$. By (4.4), this implies $\rho^{-q / p} \in L^{t}\left(d s_{1}^{2}\right)$.

Applying Hölder's inequality to the product

$$
|f|^{q}=\left(|f|^{q} \rho^{q / p}\right) \cdot\left(\rho^{-q / p}\right),
$$

we obtain

$$
\int_{S}|f|^{q} d A_{1} \leq\left(\int_{S}|f|^{s q} \rho^{s q / p} d A_{1}\right)^{1 / s}\left(\int_{S} \rho^{-t q / p} d A_{1}\right)^{1 / t} .
$$

Since $s q / p=1$, raising the above inequality to the power $1 / q$ gives us

$$
\|f\|_{L^{q}\left(d s_{1}^{2}\right)} \leq c t e \cdot\|f\|_{L^{p}\left(d s_{0}^{2}\right)} .
$$

4.3. If $u, v$ are two functions on $S$, we write $(u \mid v)_{i}:=\int_{S} u \cdot v d A_{i}$ for their scalar product in $L^{2}\left(d s_{i}^{2}\right)$. The gradient of a function $u$ with respect to $d s_{i}^{2}$ is denoted by ${ }^{i} \nabla u$. Observe that ${ }^{0} \nabla=(1 / \rho)^{1} \nabla$.

If $X$ and $Y$ are two vector fields, their pointwise scalar product with respect to $d s_{i}^{2}$ is a function denoted by $\langle X, Y\rangle_{i}$. We have $\langle X, Y\rangle_{0}=\rho\langle X, Y\rangle_{1}$. The integral of this function will be written

$$
(X \mid Y)_{i}=\int_{S}\langle X, Y\rangle_{i} d A_{i} .
$$

The Dirichlet integral of a function $u$ is

$$
\left(\left.{ }^{1} \nabla u\right|^{1} \nabla u\right)_{1}=\left(\left.{ }^{0} \nabla u\right|^{0} \nabla u\right)_{0},
$$

it depends on the conformal structure of $S$, but not on the metric, nor on the divisor. The Dirichlet integral of $u$ will be simply written $(\nabla u \mid \nabla u)$.

Let $H\left(d s_{i}^{2}\right)$ be the Sobolev space of functions $u \in L^{2}\left(d s_{i}^{2}\right)$ with finite Dirichlet inegral. This is a Hilbert space with scalar product

$$
(u \mid v)_{H\left(d s_{i}^{2}\right)}:=(u \mid v)_{i}+(\nabla u \mid \nabla v) .
$$

\subsection{Sobolev's embedding.}

Proposition 2. There exists a constant $C$ such that for all $u \in H\left(d s_{i}^{2}\right)$ and all $p \in[0, \infty[$, we have

$$
\|u\|_{L^{p}\left(d s_{i}^{2}\right)} \leq C \cdot \sqrt{p} \cdot\|u\|_{H\left(d s_{i}\right)} .
$$

Proof. By standard arguments, we reduce the proof to a local inequality which is proved in the appendix. 


\subsection{Conformal invariance of $H$.}

Proposition 3. $H\left(d s_{0}^{2}\right)=H\left(d s_{1}^{2}\right)$ (i.e., $H\left(d s_{0}^{2}\right)$ and $H\left(d s_{1}^{2}\right)$ are equal as sets and their norms define the same topology).

Proof. Since $\|u\|_{H\left(d s_{i}^{2}\right)}=(u \mid u)_{i}+(\nabla u \mid \nabla u)$, we just have to show that

$$
\|u\|_{L^{2}\left(d s_{0}^{2}\right)} \leq \text { const } \cdot\|u\|_{H\left(d s_{1}^{2}\right)}
$$

and

$$
\|u\|_{L^{2}\left(d s_{1}^{2}\right)} \leq \text { const } \cdot\|u\|_{H\left(d s_{0}^{2}\right)} .
$$

To check (i), choose $p>\max \{2,2 /(\alpha+1)\}$. By Proposition 1, we have $\|u\|_{L^{2}\left(d s_{0}^{2}\right)} \leq$ const $\cdot\|u\|_{L^{p}\left(d s_{1}^{2}\right)}$. We conclude by Proposition 2 .

The inequality (ii) is verified in the same way by choosing

$$
p>\max \{2,2(\omega+1)\} .
$$

4.6 A compactness result. The Sobolev space of functions $u \in L^{q}\left(d s_{1}^{2}\right)$ such that $\left|{ }^{1} \nabla u\right| \in L^{q}\left(d s_{1}^{2}\right)$ is denoted by $W^{1, q}\left(d s_{1}^{2}\right)$. The classical theorem of Rellich-Kondrachov (see [8]) says that there is a compact embedding

$$
W^{1, q}\left(d s_{1}^{2}\right) \subset L^{p}\left(d s_{1}^{2}\right),
$$

for all $1<q<2, p<2 q /(2-q)$. This, with Propositions 1 and 3 (and the obvious fact $\left.H\left(d s_{1}^{2}\right) \subset W^{1, q}\left(d s_{1}^{2}\right)\right)$, implies the following sharpening of Proposition 2:

Proposition 4. The embedding $H\left(d s_{i}^{2}\right) \subset L^{p}\left(d s_{i}^{2}\right)$ is compact for all $p \in[1, \infty[$.

\subsection{The Poincaré inequality.}

Proposition 5. Let $\psi \in L^{2}\left(d s_{0}^{2}\right)$ be a function such that $\int_{S} \psi d A_{0} \neq 0$, then there exists a constant $C_{1}$ such that $(u \mid u)_{0} \leq C_{1}(\nabla u \mid \nabla u)$, for all $u \in H\left(d s_{0}^{2}\right)$ such that $(u \mid \psi)_{0}=0$.

Proof. We have to show that there exists a number $\mu>0$ such that $\forall u \in$ $H\left(d s_{0}^{2}\right)$, if $(u \mid \psi)_{0}=0$ and $(u \mid u)_{0}=1$, then $(\nabla u \mid \nabla u) \geq \mu$.

To this aim, set $\mu:=\inf \left\{(\nabla u \mid \nabla u): u \in H\left(d s_{0}^{2}\right),(u \mid \psi)_{0}=0,(u \mid u)=1\right\}$. We have to show that $\mu \neq 0$.

Choose a sequence $\left\{\varphi_{j}\right\} \subset H\left(d s_{0}^{2}\right)$ such that $\left(\varphi_{j} \mid \psi\right)_{0}=0,\left(\varphi_{j} \mid \varphi_{j}\right)_{0}=1$, and $\left(\nabla \varphi_{j} \mid \nabla \varphi_{j}\right) \rightarrow \mu$. Thus, $\left\{\varphi_{j}\right\}$ is a bounded sequence in the Hilbert space $H\left(d s_{0}^{2}\right)$. We can therefore find a weakly convergent subsequence. Since $H\left(d s_{0}^{2}\right) \subset L^{2}\left(d s_{0}^{2}\right)$ is a compact inclusion, we may assume that this subsequence is strongly convergent in $L^{2}\left(d s_{0}^{2}\right)$. We still note $\left\{\varphi_{j}\right\}$ this subsequence and $\varphi_{\infty}$ its limit. We have

$$
\begin{gathered}
\left(\varphi_{\infty} \mid \psi\right)_{0}=\lim \left(\varphi_{j} \mid \psi\right)_{0}=0 ; \\
\left(\varphi_{\infty} \mid \varphi_{\infty}\right)_{0}=\lim \left(\varphi_{j} \mid \varphi_{j}\right)_{0}=1 ; \\
\left\|\varphi_{\infty}\right\|_{H\left(d s_{0}^{2}\right)} \leq \liminf \left\|\varphi_{j}\right\|_{H\left(d s_{0}^{2}\right)}=\sqrt{1+\mu} .
\end{gathered}
$$


Thus we have $\left(\nabla \varphi_{\infty} \mid \nabla \varphi_{\infty}\right) \leq \mu$. By the choice of $\mu$, we must have equality. The proof will therefore be finished if one shows that $\left(\nabla \varphi_{\infty} \mid \nabla \varphi_{\infty}\right)>0$.

Suppose $\left(\nabla \varphi_{\infty} \mid \nabla \varphi_{\infty}\right)=0$, then $\varphi_{\infty}$ must be a constant, and this constant must be 0 since $\left(\varphi_{\infty} \mid \psi\right)_{0}=0$. This is however impossible because $\left(\varphi_{\infty} \mid \varphi_{\infty}\right)_{0}=$ 1 .

\subsection{The Trudinger inequality.}

Theorem 6. There exist positive constants $\tau_{i}$ and $D_{i}$ such that for all $u \in$ $H\left(d s_{i}^{2}\right):$ if $\int_{S} u d A_{i}=0$ and $(\nabla u \mid \nabla u) \leq 1$, then

$$
\int_{S} e^{b u^{2}} d A_{i} \leq D_{i}
$$

for all $b<2 \pi \tau_{i}$.

Proof. This is a classical corollary of the Sobolev inequality (Proposition 2), see $[10$, p. 23].

Definition. The Trudinger constant of $\left(S, d s_{i}^{2}\right)$ is the number

$$
\begin{aligned}
& \tau\left(S, d s_{i}^{2}\right):=\sup \left\{\tau: \int_{S} e^{2 \pi \tau u^{2}} d A_{i} \text { is bounded for } u \in H\left(d s_{i}^{2}\right),\right. \\
& \left.\qquad \int_{S} u d A_{i}=0,(\nabla u \mid \nabla u) \leq 1\right\} .
\end{aligned}
$$

Remark. The value of $\tau\left(S, d s_{i}^{2}\right)$ given by the proof above is only a crude estimate. The best value will be given in the next subsection. Set $b_{i}:=\tau\left(S, d s_{i}^{2}\right)$. Corollary 7. Fix $\delta \in \mathbf{R}, p>1$ and $\varphi \in L^{p}\left(d s_{i}^{2}\right)$. Then for all $v \in H\left(d s_{i}^{2}\right)$ such that $\int_{S} v d A_{i}=0$ and $b<b_{i}$, we have

$$
\left|\left(e^{\delta v} \mid \varphi\right)\right| \leq D_{i}^{1 / q} \cdot\|\varphi\|_{L^{p}\left(d s_{i}^{2}\right)} \cdot \exp \left(\frac{q \delta^{2}}{4 b}(\nabla v \mid \nabla v)\right),
$$

where $q:=p /(p-1)$.

Proof. We can imitate the proof of inequality (3.5) in [10].

To extend this inequality to the case where $\int_{S} u d A_{i}$ does not necessarily vanish, we introduce the following functional defined on $H\left(d s_{i}^{2}\right)$ :

$$
I(u)=I_{b, q, \delta, \psi}(u):=\frac{q \delta^{2}}{4 b}(\nabla u \mid \nabla u)+\delta(\psi \mid u) .
$$

Theorem 8. Let $\delta>0, p>1$ and $\psi \in L_{i}^{2}$ such that $\int_{S} \psi d A_{0}=1$. Then there exists a constant $D_{i}^{\prime}$ such that for all $\varphi \in L^{p}\left(d s_{i}^{2}\right), b<b_{i}$ and $u \in H\left(d s_{i}^{2}\right)$ we have

$$
\left|\left(e^{\delta u} \mid \varphi\right)\right| \leq D_{i}^{\prime} \cdot\|\varphi\|_{L^{p}\left(d s_{i}^{2}\right)} \cdot \exp (I(u)),
$$

where $I(u)=I_{b, q, \delta \psi}(u)$ is defined above and $q=p /(p-1)$.

Proof. Set $\bar{u}=\left(\int_{S} u d A_{i}\right) /\left(\int_{S} d A_{i}\right)$. 
Case 1. $\bar{u}=0$. We have by Poincaré inequality (Proposition 5)

$$
(u \mid \psi)^{2} \leq(u \mid u)(\psi \mid \psi) \leq C(\nabla u \mid \nabla u)(\psi \mid \psi),
$$

and thus (since $x^{2} \leq y^{2} \Rightarrow y \geq-\sqrt{x^{2}}$ ),

$$
(u \mid \psi) \geq-\sqrt{C(\psi \mid \psi)} \sqrt{(\nabla u \mid \nabla u)} .
$$

Choose $b^{\prime}$ such that $b<b^{\prime}<b_{i}$ and set $\mu=\left(q \delta^{2}\right) /(4 b), \mu^{\prime}=\left(q \delta^{2}\right) /\left(4 b^{\prime}\right)$, and $\vartheta=\delta^{2} C(\psi \mid \psi) / 4\left(\mu-\mu^{\prime}\right)$.

We have $\mu>\mu^{\prime}, \vartheta>0$, and from $\left(\sqrt{\left(\mu-\mu^{\prime}\right)(\nabla u \mid \nabla u)}-\sqrt{\vartheta}\right)^{2}>0$, we deduce

$$
\mu(\nabla u \mid \nabla u)-\delta \sqrt{C(\psi \mid \psi)} \sqrt{(\nabla u \mid \nabla u)} \geq \mu^{\prime}(\nabla u \mid \nabla u)-\vartheta .
$$

The inequalities (i) and (ii) imply

$$
I(u)+\vartheta=\mu(\nabla u \mid \nabla u)+\delta(u \mid \psi)+\vartheta \geq \mu^{\prime}(\nabla u \mid \nabla u) .
$$

Combining this inequality with Corollary 7 , we obtain

$$
\left(e^{\delta u} \mid \varphi\right) \leq D^{1 / q}\|\varphi\|_{L^{p}\left(d s_{i}^{2}\right)} \cdot e^{\mu^{\prime}(\nabla u \mid \nabla u)} \leq\left(D_{i}^{1 / q} e^{\vartheta}\right)\|\varphi\|_{L^{p}\left(d s_{i}^{2}\right)} \cdot e^{I(u)} .
$$

Case 2. $\bar{u} \neq 0$. The fact that $(1 \mid \psi)=1$ implies $e^{\bar{u}}=e^{(\bar{u} \mid \psi)}$. Set $w:=u-\bar{u}$, we have

$$
\left(e^{\delta u} \mid \varphi\right)=e^{\delta \bar{u}} \cdot\left(e^{\delta w} \mid \varphi\right)=e^{\delta(\bar{u} \mid \psi)}\left(e^{\delta w} \mid \varphi\right) .
$$

Since $\int_{S} w d A_{i}=0$, we conclude using Case 1 .

4.9 The Trudinger constant. It will be important in applications to know the actual value of the Trudinger constant (the least upper bound of the $\tau$ such that the Trudinger inequality $\int_{S} e^{b u^{2}} d A \leq D$ holds for all $\left.b<2 \pi \cdot \tau\right)$.

In the smooth case, this value is known from the work of Moser [12] and Cherrier [4]. They proved that $\tau\left(S, d s_{1}^{2}\right)=2$.

Theorem 9 (Moser-Cherrier). There exists a constant $D_{1}$ such that if $u \in H\left(d s_{1}^{2}\right)$ satisfies $\int_{S} u d A_{1}=0$ and $(\nabla u \mid \nabla u) \leq 1$, then

$$
\int_{S} e^{4 \pi u^{2}} d A_{1} \leq D_{1} .
$$
B .)

(Recall that $d s_{1}^{2}$ is a smooth metric on $S$, whereas $d s_{0}^{2}$ represents the divisor

In the presence of conical singularities, the Trudinger constant has a different value:

Corollary 10. We have $\tau\left(S, d s_{0}^{2}\right)=\min \{2,2+2 \alpha\}$, i.e., the Trudinger inequality $\int_{S} e^{b u^{2}} d A_{0} \leq D_{0}$ holds for all $b<2 \pi \cdot \min \{2,2+2 \alpha\}$.

(Recall that $\alpha=\min _{i}\left\{\beta_{i}\right\}$.) Since this number depends only on $\boldsymbol{\beta}$, we will write $\tau(S, \boldsymbol{\beta}):=\min \{2,2+2 \alpha\}$. 
Proof. For $u \in H\left(d s_{0}^{2}\right)$, we will write $\bar{u}:=\frac{1}{A_{0}} \int_{S} u d A_{0}$ and $\tilde{u}:=\frac{1}{A_{1}} \int_{S} u d A_{1}$ (where $A_{i}:=\int_{S} d A_{i}$ is the area of $\left(S, d s_{i}^{2}\right)$ ). Observe that by Proposition 1,

$$
|\tilde{u}| \leq \frac{1}{A_{1}}\|u\|_{L^{1}\left(d s_{1}^{2}\right)} \leq \frac{1}{A_{1}}\|u\|_{L^{r}\left(d s_{0}^{2}\right)}
$$

for $r>\max \{1, \omega+1\}$. Combining this inequality with Proposition 2, we obtain:

$$
|\tilde{u}| \leq c t e^{\prime}\|u\|_{H\left(d s_{0}^{2}\right)} .
$$

Set $B:=\left\{u \in H\left(d s_{0}^{2}\right): \bar{u}=0,(\nabla u \mid \nabla u) \leq 1\right\}$, we deduce from Poincaré and the above inequalities that there exists an absolute constant $\tilde{c}$ such that $|\tilde{u}| \leq \tilde{c}$, for all $u \in B$.

Let $b<2 \pi \tau$, we have to prove that $\int_{s} e^{b u^{2}} d A_{0}$ is bounded for $u \in B$. To this aim set $u_{1}:=u-\tilde{u}$, we then have

$$
\int_{S} e^{b u^{2}} \cdot d A_{0}=e^{b \tilde{u}^{2}} \cdot \int_{S} e^{b u_{1}^{2}} \cdot e^{2 b \tilde{u} u_{1}} \cdot d A_{0} \leq e^{b \tilde{c}^{2}} \cdot \int_{S} e^{b u_{1}^{2}} \cdot e^{2 b \tilde{c} u_{1}} \cdot d A_{0} .
$$

Now choose $q, p, s \in \mathbf{R}$ such that $1<q<p \tau / 2<2 \pi \tau / b$ and $\frac{1}{q}+\frac{1}{s}=1$. Hölder's inequality tells us that

$$
\int_{S} e^{b u^{2}} \cdot d A_{0} \leq\left(e^{b \tilde{c}^{2}}\right) \cdot\left(\int_{S} e^{q b u_{1}^{2}} d A_{0}\right)^{1 / q} \cdot\left(\int_{S} e^{s 2 b \tilde{c} u_{1}} d A_{0}\right)^{1 / s} .
$$

Setting $\delta:=2 s b \tilde{c}$, we know by Corollary 7 that

$$
\int_{S} e^{\delta u_{1}} d A_{0}=\int_{S} e^{\delta u_{1}} \rho d A_{1}
$$

is bounded.

Since $p>\max \{q, q /(\alpha+1)\}$, Proposition 1 gives

$$
\left(\int_{S} e^{q b u_{1}^{2}} d A_{0}\right)^{1 / q} \leq c t e \cdot\left(\int_{S} e^{p b u_{1}^{2}} d A_{1}\right)^{1 / p} .
$$

By Theorem 9, this last integral is bounded since $b p<4 \pi, \int_{S} u d A_{1}=0$ and $(\nabla u \mid \nabla u) \leq 1$.

Remark. It is not clear from our proof of Corollary 10 whether the Trudinger inequality holds for $b=2 \pi \tau(S, \boldsymbol{\beta})$. However, some extra work (e.g., using the symmetrization procedure used by Moser in his proof of Theorem 9) would show that this is indeed the case. Since we will not need it, we omit this point.

4.10 Some (classical) consequences of the Trudinger inequality. By Theorem 8 , we know that $e^{u} \in L^{p}\left(d s_{i}^{2}\right)$ for all $p<\infty$, if $u \in H\left(d s_{i}^{2}\right)$. More precisely, we have

Proposition 11. The embedding

$$
\begin{aligned}
H\left(d s_{1}^{2}\right) & \subset L^{p}\left(d s_{i}^{2}\right) \\
u & \rightarrow e^{\delta u}
\end{aligned}
$$

is compact for all $p<\infty$. 
Proof. Let $\left\{u_{j}\right\}$ be a bounded sequence in $H\left(d s_{1}^{2}\right)$ and $p<\infty$. We have to show that $\left\{u_{j}\right\}$ has a convergent subsequence in $L^{p}\left(d s_{1}^{2}\right)$.

Choose $q, r$ such that $2 p /(2+p)<q<2$ and $\frac{1}{r}+\frac{1}{2}=\frac{1}{q}$.

Theorem 8 implies that $\left\{e^{\delta u_{j}}\right\}$ is bounded in $L^{s}\left(d s_{1}^{2}\right)$ (for all $s$ ). On the other hand, we have (using Hölder's inequality)

$$
\begin{aligned}
\left\|^{1} \nabla e^{\delta u_{j}}\right\|_{L^{q}\left(d s_{1}^{2}\right)} & =\delta\left\|\left.e^{\delta u_{j}}\right|^{1} \nabla u_{j} \mid\right\|_{L^{q}\left(d s_{1}^{2}\right)} \\
& \leq \delta\left\|e^{\delta u_{j}}\right\|_{L^{r}\left(d s_{1}^{2}\right)} \cdot\left\|u_{j}\right\|_{L^{2}\left(d s_{1}^{2}\right)} .
\end{aligned}
$$

Thus $\left\{e^{\delta u_{j}}\right\}$ is a bounded sequence in $W^{1, q}\left(d s_{1}^{2}\right)$. We conclude by RellichKondrachov (since $p<2 q /(2-q)$ ).

Corollary 12. The embedding

$$
\begin{gathered}
H\left(d s_{0}^{2}\right) \subset L^{p}\left(d s_{0}^{2}\right) \\
u \rightarrow e^{\delta u}
\end{gathered}
$$

is compact for all $p<\infty$.

The proof is obvious from Propositions 1, 3, and 11.

Corollary 13. Let $\varphi \in L^{q}\left(d s_{0}^{2}\right) \quad(q>2), \delta \in \mathbf{R}$. Then the function

$$
\begin{aligned}
& H\left(d s_{0}^{2}\right) \rightarrow \mathbf{R} \\
& u \rightarrow\left(e^{\delta u} \mid \varphi\right)
\end{aligned}
$$

is continuous for the weak topology in $H\left(d s_{0}^{2}\right)$.

Proof. Let $p:=q /(q-1)(<2)$ and let $\left\{u_{j}\right\}$ be a sequence in $H\left(d s_{0}^{2}\right)$ weakly converging to $u^{*}$. Then $\left\{e^{\delta u_{j}}\right\}$ strongly converges to $e^{\delta u^{*}}$ in the $L^{p}\left(d s_{0}^{2}\right)$ topology. This proves our statement since the pairing

$$
\begin{gathered}
L^{p}\left(d s_{0}^{2}\right) \times L^{q}\left(d s_{0}^{2}\right) \rightarrow \mathbf{R} \\
(f, g) \rightarrow(f \mid g)
\end{gathered}
$$

is a continuous function.

$$
\text { 5. THE EQUATION } \Delta u=h e^{\delta u}-h_{0}
$$

As in the preceding section, $d s_{0}^{2}$ is a conformal metric with conical singularities representing a divisor $\boldsymbol{\beta}$ on a compact generalized Riemann surface $S$ without boundary. In this section we study the equation

$$
\Delta u=h e^{\delta u}-h_{0},
$$


where $h$ and $h_{0}$ are two Hölder continuous functions on $S, \delta$ is a positive number, and $\Delta$ is the Laplacian corresponding to the metric $d s_{0}^{2}$.

We denote by $(u \mid v)$ the $L^{2}$-scalar product with respect to $d s_{0}^{2}$, and by $H$ the Sobolev space of functions with $(u \mid u)+(\nabla u \mid \nabla u)<\infty$. We will also use the subspace $H^{\prime}:=\{u \in H: \bar{u}=0\}$ (where $\bar{u}:=(u \mid 1) /(1 \mid 1)$ is the average of $\left.u\right)$.

We now introduce two functionals $\mathscr{F}$ and $\mathscr{G}: H \rightarrow \mathbf{R}$ defined by

$$
\mathscr{F}(u):=(\nabla u \mid \nabla u)+2\left(h_{0} \mid u\right), \quad \mathscr{G}(u):=\left(h \mid e^{\delta u}\right) .
$$

The derivatives of these are

$$
D \mathscr{F}(u)(v)=2\left(\Delta u+h_{0} \mid v\right), \quad D \mathscr{G}(u)(v)=\delta\left(h e^{\delta u} \mid v\right) .
$$

Finally, we define $\gamma:=\left(h_{0} \mid 1\right)=\int_{S} h_{0} d A$.

5.1 Basic properties of $\mathscr{F}$ and $\mathscr{G}$. (a) The functional $\mathscr{G}$ is continuous with respect to the weak topology in $H$.

This is Corollary 13 in $\S 4$.

(b) There exists a constant $c$ such that $\left|\left(h_{0} \mid u\right)\right| \leq c \cdot \sqrt{(\nabla u \mid \nabla u)}$ for all $u \in H^{\prime}$. Indeed, $\left(h_{0} \mid u\right)^{2} \leq\left(h_{0} \mid h_{0}\right)(u \mid u) \leq C_{1}\left(h_{0} \mid h_{0}\right)(\nabla u \mid \nabla u)$ by Poincaré inequality.

(c) The functional $\mathscr{F}$ is bounded below on $H^{\prime}$.

For, we have

$$
\mathscr{F}(u)=(\nabla u \mid \nabla u)+2\left(h_{0} \mid u\right) \geq(\nabla u \mid \nabla u)-2 c \cdot \sqrt{(\nabla u \mid \nabla u)} \geq-c^{2} .
$$

(d) $\mathscr{F}$ is coercive in $H^{\prime}$ (i.e., if $B^{\prime}$ is a subset of $H^{\prime}$, then $\mathscr{F}$ is bounded on $B^{\prime}$ if and only if $B^{\prime} \subset H^{\prime}$ is bounded).

Thanks to (c) and Poincaré's inequality, we only have to show that $\mathscr{F}$ is bounded above: $\mathscr{F}(u)=(\nabla u \mid \nabla u)+2\left(h_{0} \mid u\right) \leq(\nabla u+\nabla u)+2 c \cdot \sqrt{(\nabla u \mid \nabla u)} \leq$ $\|u\|_{H}+2 c \sqrt{\|u\|_{H}}$.

(e) If $h \not \equiv 0$, then $\mathscr{G}$ has no critical points.

This is obvious from the computation of $D \mathscr{G}$.

5.2 Admissible subspaces. Before solving (5.1), we have to choose the function space in which we want to seek a solution. Observe first of all that if $u$ satisfies (5.1), then $\mathscr{G}(u)=\gamma \quad\left(:=\left(h_{0} \mid 1\right)\right)$ (this comes from $\left.\int_{S} \Delta u d A=-\int_{S} d * d u=0\right)$. This condition is called the constraint on $u$.

Definition. A linear subspace $\widetilde{H} \subset H$ is admissible (with respect to the constraint $\mathscr{G}(u)=\gamma$ ) if it is closed and if there exists a complementary subspace $L \subset H$ such that $H=L+\widetilde{H}$ (direct sum) and the following condition is satisfied: if $u \in\{u \in \widetilde{H}: \mathscr{G}(u)=\gamma\}$ and $v \in L$, then we have

$$
(\nabla u \mid \nabla v)=\left(h e^{\delta u} \mid v\right)=\left(h_{0} \mid v\right)=0 .
$$

Examples. The space $H$ itself is admissible. The intersection of two admissible subspaces is admissible. The sum of two admissible subspaces is admissible. If $\gamma=0$, then $H^{\prime}$ is admissible (take $L$ to be the constant functions). If $G$ is a 
group of isometries of $\left(S, d s_{0}^{2}\right)$ such that $h$ and $h_{0}$ are $G$-invariant, then the subspace $H^{G}:=\{u \in H: u \circ g=u \forall g \in G\}$ of invariant functions is admissible (choose $L$ to be the orthogonal complement of $H^{G}$ ).

5.3 The variational method. Equation (5.1) has been mainly studied by the variational method, which is based on the following.

Theorem 1. If there exists an admissible subspace $\widetilde{H} \subset H$ and a number $m \in \mathbf{R}$ such that

(i) The set $B:=\{u \in \widetilde{H}: \mathscr{G}(u)=\gamma$ and $\mathscr{F}(u) \leq m\}$ is not empty;

(ii) $B$ is a bounded subset of $H$.

Then there exists a number $\lambda \in \mathbf{R}$ and a function $u \in \widetilde{H} \cap C^{0}(S)$ such that $u$ is of class $C^{2}$ in $S \backslash \operatorname{supp}(\boldsymbol{\beta}), \mathscr{G}(u)=\gamma$ and $u$ satisfies the equation

$$
\Delta u=\lambda h e^{\delta u}-h_{0} .
$$

Remark. If $\gamma \neq 0$ and if there is a solution $u$ of $\left(5.1_{\lambda}\right)$ such that $\mathscr{G}(u)=\gamma$, then $\lambda=1$ (this is obvious upon integrating $\left(5.1_{\lambda}\right)$ ).

Proof. Set $\mu:=\inf \{\mathscr{F}(u): u \in B\} \quad(=\inf \{\mathscr{F}(u): u \in \widetilde{H}$ and $\mathscr{G}(u)=\gamma\})$.

First step. $\mu$ is achieved. This follows by standard arguments from the weak continuity of $\mathscr{G}$, the weak semicontinuity of $\mathscr{F}$ and the compactness of the inclusion $\widetilde{H} \subset L^{2}$.

Second step. $u^{*}$ is a weak solution of equation $\left(5.1_{\lambda}\right)$. For $\left(5.1_{\lambda}\right)$ is the Euler-Lagrange equation of the variational problem defining $\mu$.

Third step. $u^{*}$ is Hölder-continuous. The metric is locally given by $d s_{0}^{2}=$ $\rho(z)|z|^{2 \beta}|d z|^{2}$ (with $\rho$ continuous), therefore, equation $\left(5.1_{\lambda}\right)$ has the local form

$$
-\left(\partial^{2} / \partial x^{2}+\partial^{2} / \partial y^{2}\right) u^{*}=\rho(z)|z|^{2 \beta}\left(\lambda h e^{\delta u^{*}}-h_{0}\right) .
$$

Now, recall (\$4.8) that $e^{\delta u} \subset L^{q}$ for all $q<\infty$, hence the right-hand side of the above equation belongs to $L^{p}$ for some number $p>1$, and we can apply the $L^{p}$-regularity theory of the classical Laplace operator:

Theorem [7, Proposition 6]. Let $\Omega$ be some domain in $\mathbf{R}^{n}$ and $g \in L^{p}(\Omega)$ for some number $p>n / 2$. Then any weak solution of

$$
u \in W^{1,2}(\Omega), \quad-\sum_{i=1}^{n} \frac{\partial^{2}}{\partial x_{i}^{2}} u=g
$$

is locally Hölder-continuous.

Fourth step. $u^{*}$ is $C^{2}$ off the singularities and $\left(5.1_{\lambda}\right)$ is satisfied in the strong sense. Since $u^{*}$ is Hölder-continuous, the right-hand side of equation (5.2) is Hölder-continuous and we can apply the Hölder-regularity theory for the classical Laplacian: 
Theorem ([8, Theorem 6.13] or [7, Proposition 9]). Let $\Omega$ be a domain with smooth boundary in $\mathbf{R}^{n}$. If $u, g \in C^{k, \alpha}(\Omega)$ verify

$$
-\sum_{i=1}^{n} \frac{\partial^{2}}{\partial x_{i}^{2}} u=g
$$

in the weak sense. Then $u \in C^{k+2, \alpha}(\Omega)$ and the equation above is in fact satisfied in the strong sense.

Therefore $u^{*} \in C^{2}(S \backslash \operatorname{supp}(\boldsymbol{\beta}))$. Now $u^{*}$ also satisfies $u^{*} \in \widetilde{H}$ and $\mathscr{G}\left(u^{*}\right)=$ $\gamma$, hence Theorem 1 is proved.

5.4 Solving (5.1) when $h \equiv 0$. In this case, equation (5.1) is linear (it is the classical Poisson equation).

Theorem 2. If $h \equiv 0$, then (5.1) is solvable if and only if $\gamma=0$. The solution is unique up to an additive constant, it is continuous and $C^{2}$ in $S \backslash \operatorname{supp}(\boldsymbol{\beta})$.

Proof. The difference between two solutions satisfies $\Delta w=0$. Uniqueness follows.

If $(5.1)$ has a solution $u$, then $\gamma=\left(h_{0} \mid 1\right)=(-\Delta u \mid 1)=0$.

Conversely, assume $\gamma=0$, and set $\widetilde{H}=H^{\prime} \quad(=\{u \in H:(u \mid 1)=0\})$, $m:=\mathscr{F}(0)=0$. We have then

(i) $B:=\left\{u \in H^{\prime}: \mathscr{F}(u) \leq 0\right\} \neq \varnothing$;

(ii) $\mathscr{F}$ is bounded below on $B$ (see $5.1 \mathrm{c}$ );

(iii) $B$ is bounded in $H$ (follows from 5.1d).

We conclude the proof by Theorem 1 .

5.5 Solving (5.1) when $\gamma=0$ and $h \not \equiv 0$. Let $f$ be a function such that $\Delta f+h_{0}=0$ (known to exist by Theorem 2).

Theorem 3. If $\gamma=0$ and $h \not \equiv 0$, then (5.1) has a solution if and only if the following two conditions are verified:

(1) $h$ changes sign;

(ii) $\left(h \mid e^{\delta f}\right)<0$.

All solutions are continuous and $C^{2}$ in $S \backslash \operatorname{supp}(\boldsymbol{\beta})$.

Proof. First, we show that conditions (1) and (2) are necessary. Let $u$ be a solution of (5.1). Integrating (5.1) gives $\mathscr{G}(u)=\left(h e^{\delta u} \mid 1\right)=\gamma=0$, hence $h$ must change sign. Set $w:=u-f$, we have:

$$
\left(h \mid e^{\delta f}\right)=\left(e^{-\delta w} \mid h e^{\delta u}\right)=\left(e^{-\delta w} \mid \Delta w\right)=-\delta\left(e^{-\delta w} \nabla w \mid \nabla w\right)<0
$$

(since $w \not \equiv 0)$.

Now we show that these conditions are sufficient: Let $\widetilde{H}:=\left\{u \in H:\left(u \mid e^{\delta f}\right)\right.$ $=0\}$ and $L \subset H$ be the set of constant functions $(\cong \mathbf{R})$, observe that $\widetilde{H}$ is admissible.

Since $h$ changes sign, we can find a function $v \in C^{1}(S)$ such that $\mathscr{G}(v)=0$. Adding a constant if necessary, we may assume $v \in \widetilde{H}$. Set $m:=\mathscr{F}(v)$ and 
$B:=\{u \in \widetilde{H}: \mathscr{G}(u)=0$ and $\mathscr{F}(u) \leq m\}$. Poincaré's inequality ( $\S 4.7)$ tells us that $(u \mid u) \leq C_{1}(\nabla u \mid \nabla u)$ for all $u \in \widetilde{H}$, hence we easily show (using 5.1c and $5.1 \mathrm{~d})$ that $\mathscr{F}$ is bounded below on $B$ and that $B$ is a bounded subset in $H$.

Theorem 1 tells us then that $\left(5.1_{\lambda}\right)$ has a solution for some $\lambda$. We claim that $\lambda>0$. Indeed, let $w:=u-f$ where $u$ solves $\left(5.1_{\lambda}\right)$, then $\left(\lambda h \mid e^{\delta f}\right)=$ $\left(e^{-\delta w} \mid \Delta w\right)=-\delta\left(e^{-\delta w} \nabla w \mid \nabla w\right)<0$. The condition (2) implies $\lambda>0$.

We directly check that $u^{\prime}:=u+\log (\lambda) / \delta$ is a solution of $(5.1)$.

5.6 Solving (5.1) when $\gamma<0$. An obvious necessary condition to solve (5.1) when $\gamma<0$ is: $\inf (h)<0$. This condition is however not sufficient, a fine study (in the smooth case) has been carried out by Kazdan and Warner (see $[10, \S 10])$. Our goal here is more modest and we solve (5.1) under the stronger assumption $\sup (h)<0$.

Theorem 4. Suppose $\gamma<0$ and $\sup (h)<0$. Then there exists a unique solution of (5.1). This solution is continuous and $C^{2}$ on $S \backslash \operatorname{supp}(\boldsymbol{\beta})$.

Proof. Suppose we had two solutions $u$ and $v$ of (5.1), then their difference $w$ would satisfy $\Delta w=e^{\delta v} h\left(e^{\delta w}-1\right)$. Thus $w \Delta w \leq 0$, but $\int_{S} w \Delta w d A=$ $(\nabla w \mid \nabla w) \geq 0$. Therefore $w$ must be constant, since $\mathscr{G}(u)=\mathscr{G}(v)=\gamma \neq 0$, this constant is 0 and $u=v$.

Let us now show the existence of a solution: since $h, \gamma<0$, we can find a constant $c \in \mathbf{R}$ such that $\mathscr{G}(c)=\gamma$. Set $\widetilde{H}=H$ and $m:=\mathscr{F}(c)$. Then $B:=\{u \in H: \mathscr{G}(u)=\gamma, \mathscr{F}(u) \leq m\} \neq \varnothing$.

Lemma. $|\mathscr{G}|$ is bounded below on $H^{\prime}$ by a positive constart.

(Proof. We have for all $u^{\prime} \in H^{\prime}:\left|\mathscr{G}\left(u^{\prime}\right)\right| \geq \inf |h| \cdot\left(e^{\delta u^{\prime}} \mid 1\right) \geq \inf |h|\left(1+\delta u^{\prime} \mid 1\right)$ $=\inf |h| \cdot(1 \mid 1)>0$.)

Corollary. Set $\bar{u}:=(u \mid 1) /(1 \mid 1)$. Then $\bar{u}$ is bounded on $B$.

(Proof. $u=u^{\prime}+\bar{u}$ and we have $\mathscr{G}(u)=e^{\delta \bar{u}} \mathscr{G}\left(u^{\prime}\right)$. Hence the lemma implies that $\bar{u}$ is bounded above on $B$. On the other hand, using $\S 5.1 \mathrm{c}$ and $\gamma<0$, we see that $\bar{u}=\frac{1}{2 \gamma}\left(\mathscr{F}(u)-\mathscr{F}\left(u^{\prime}\right)\right)$ is bounded below on $B$.)

To finish the proof of Theorem 4, we have to check that conditions (i), (ii) and (iii) of Theorem 1 are satisfied:

(i) $B$ is not empty (obvious).

(ii) $\mathscr{F}$ is bounded below on $B$ : Indeed $\mathscr{F}(u)=\mathscr{F}\left(u^{\prime}+\bar{u}\right)=\mathscr{F}\left(u^{\prime}\right)+2 \gamma \bar{u}$ is bounded below by the above corollary and $5.1 \mathrm{c}$.

(iii) $B$ is bounded in $H$ : Set $B^{\prime}:=\{u-\bar{u}: u \in B\} \subset H^{\prime}$, then $\mathscr{F}\left(u^{\prime}\right)=$ $\mathscr{F}\left(u^{\prime}+\bar{u}\right)-2 \gamma \bar{u}$ is bounded above for $u^{\prime} \in B^{\prime}$, hence, 5.1d implies that $\left(\nabla u^{\prime} \mid \nabla u^{\prime}\right)$ is bounded on $B^{\prime}$. We have then for $u=u^{\prime}+\bar{u} \in B$

$$
\begin{aligned}
\|u\|_{H}^{2} & =\left(u^{\prime} \mid u^{\prime}\right)+\bar{u}^{2}(1 \mid 1)+\left(\nabla u^{\prime} \mid \nabla u^{\prime}\right) \\
& \leq\left(1+C_{1}\right)\left(\nabla u^{\prime} \mid \nabla u^{\prime}\right)+\bar{u}^{2}(1 \mid 1),
\end{aligned}
$$

which is bounded. 
5.7 Solving (5.1) when $\gamma>0$. An obvious and necessary condition to solve (5.1) when $\gamma>0$ is: $\sup (h)>0$. Again, this condition is not sufficient, see [3]. The solvability of (5.1) depends on the value of $\delta \gamma$ :

Theorem 5. Suppose that the conditions

(1) $\sup (h)>0$,

(2) $0<\delta \gamma<4 \pi \tau$,

(where $\tau=\min _{i}\left\{2,2+2 \beta_{i}\right\}$ is the Trudinger constant of $(S, \boldsymbol{\beta})$ ) are satisfied. Then there exists a continuous solution $u$ of equation (5.1), furthermore, $u$ is $C^{2}$ on $S \backslash \operatorname{supp}(\boldsymbol{\beta})$.

Proof. We can clearly find a function $v \in C^{1}(S)$ such that $\mathscr{G}(v)=\gamma$. Set $m:=\mathscr{F}(v)$ and $\widetilde{H}=H$, then

(i) $B:=\{u \in H: \mathscr{G}(u)=\gamma$ and $\mathscr{F}(u)<m\} \neq \varnothing$.

Now choose $b$ such that $\delta \gamma / 2<b<2 \pi \tau$ and set

$$
I(u)=\frac{\delta^{2}}{4 b}(\nabla u \mid \nabla u)+\frac{\delta}{\gamma}\left(h_{0} \mid u\right) .
$$

By Theorem 8 in $\S 4.8$ we have for $u \in B$ :

$$
\gamma=\left(e^{\delta u} \mid h\right) \leq D^{\prime} \cdot \sup (h) \cdot \exp (I(u)),
$$

and thus $I(u) \geq$ const. But we have

$$
\frac{\delta}{2 \gamma} \mathscr{F}(u)=I(u)+\frac{\delta}{2}\left(\frac{1}{\gamma}-\frac{\delta}{2 b}\right)(\nabla u \mid \nabla u)>I(u) \geq \text { const } .
$$

Thus

(ii) $\mathscr{F}$ is bounded below on $B$.

It follows from the above inequality that $(\nabla u \mid \nabla u)$ is bounded on $B$. On the other hand, if $u=u^{\prime}+\bar{u}\left(u^{\prime} \in H^{\prime}\right)$, then $2 \gamma \bar{u}=\mathscr{F}(u)-(\nabla u \mid \nabla u)-2\left(h_{0} \mid u^{\prime}\right)$, but Poincaré inequality tells us that

$$
\left(h_{0} \mid u^{\prime}\right)^{2} \leq\left(h_{0} \mid h_{0}\right)\left(u^{\prime} \mid u^{\prime}\right) \leq C_{1}\left(h_{0} \mid h_{0}\right)(\nabla u \mid \nabla u) .
$$

Hence $|\bar{u}|$ is bounded on $B$. Thus

$$
\begin{aligned}
\|u\|_{H}^{2} & =(u \mid u)+(\nabla u \mid \nabla u)=\left(u^{\prime} \mid u^{\prime}\right)+\bar{u}^{2}(1 \mid 1)+(\nabla u \mid \nabla u) \\
& \leq\left(1+C_{1}\right)(\nabla u \mid \nabla u)+\bar{u}^{2}(1 \mid 1)
\end{aligned}
$$

is bounded on $B$; hence

(iii) $B$ is bounded in $H$.

We conclude by Theorem 1 .

5.8 Proof of the main theorems. The proof of Theorems 1, 3, and 4, and of Proposition 2 in $\S 3$ are almost finished. We will complete the proofs of these results simultaneously. 
First we assume $\partial S=\varnothing$. We are thus given a divisor $\boldsymbol{\beta}$ on a compact G.R.S. $S$ without boundary and a Hölder-continuous function $K: S \rightarrow \mathbf{R}$ in such a way that the relevant hypotheses are satisfied. Choose some conformal metric $d s_{0}^{2}$ representing $\boldsymbol{\beta}$ (such a metric is easily constructed e.g., by a partition of unity). Using Theorem 2, 3, 4 or 5 in $\S 5$, we get a solution of

$$
\Delta u=K e^{2 u}-K_{0},
$$

(where $\Delta$ and $K_{0}$ denote the Laplacian and curvature of $d s_{0}$ ). The desired metric is $d s^{2}:=e^{2 u} d s_{0}^{2}$. When $K \leq 0$, we also have uniqueness from $\S 5.4$ and $\S 5.6$.

If $\partial S \neq \varnothing$, we introduce the canonical double cover $f: S^{\prime} \rightarrow S$ of $S$. Recall that $S^{\prime}$ is a compact G.R.S. with a conformal involution $\sigma: S^{\prime} \rightarrow S^{\prime}$, the surface $S$ can be identified with $S^{\prime} / \sigma$. On $S^{\prime}$ we have the divisor $\boldsymbol{\beta}^{\prime}=f^{*} \boldsymbol{\beta}$ which is invariant under $\sigma$.

Choose on $S^{\prime}$ a conformal metric $d s_{0}^{\prime}$ representing $\boldsymbol{\beta}^{\prime}$ and invariant under $\sigma$. By Riemann-Hurwitz (Corollary 2 in $\S 2$ ), we have $\chi\left(S^{\prime} \boldsymbol{\beta}^{\prime}\right)=2 \chi(S, \boldsymbol{\beta})$. Thus if $S$ and $K: S \rightarrow \mathbf{R}$ satisfy one of the hypotheses of Theorems 1,3 , or 4, or Proposition 2 in $\S 3$, then so do $S^{\prime}$ and $K^{\prime}:=K \circ f: S^{\prime} \rightarrow \mathbf{R}$. Hence, applying Theorem $2,3,4$, or 5 , in $\S 5$, we get a solution of $\Delta u^{\prime}=K^{\prime} e^{2 u^{\prime}}-K_{0}^{\prime}$, on $S^{\prime}$. Furthermore, since the space of $\sigma$-invariant functions is admissible, Theorem 1 in $\S 5$ implies that the solution can be chosen $\sigma$-invariant.

We thus obtain a conformal $\sigma$-invariant metric $d s^{\prime 2}=e^{2 u^{\prime}} d s_{0}^{\prime 2}$ of curvature $K^{\prime}$ on $S^{\prime}$ which gives the desired metric on $S \cong S^{\prime} / \sigma$.

\section{APPENDIX: A Weighted SoboleV INEQUALITY}

Proposition 1. Let $\Omega$ be some domain in $\mathbf{R}^{n}$ and $\beta>-1$, then there exists $a$ constant $C(\Omega)$, independent of $p$ such that for all $u \in C_{0}^{1}(\Omega)$,

$$
\left(\int_{\Omega}|u|^{p} \cdot|x|^{n \beta} \cdot d x\right)^{1 / p} \leq C(\Omega) \cdot p^{(n-1) / n} \cdot\left(\int_{\Omega}|D u|^{n} \cdot d x\right)^{1 / n},
$$

where $d x$ is the Lebesgue measure in $\mathbf{R}^{n}$ and $D u=\left(\partial u / \partial x_{1}, \partial u / \partial x_{2}, \ldots\right.$, $\left.\partial u / \partial x_{n}\right)$ is the euclidean gradient of $u$.

Proof. It is enough to prove the proposition for $\Omega=B:=\left\{x \in \mathbf{R}^{n}:|x|<1\right\}$ and $p>n$.

Let us introduce the operator $T$ defined by

$$
T w(x):=\frac{1}{\omega_{n-1}} \int_{B} \frac{w(y) d y}{|x-y|^{n-1}},
$$

(where $\omega_{n-1}$ is the volume of $\partial B$ ). It is easy to see that

$$
|u(x)| \leq T(|D u|) .
$$

First case. $\beta \geq 0$. Set $s:=n p /((n-1) p+n)$, then $s<\frac{n}{n-1}$ and we have $1=\frac{1}{p}+\left(\frac{1}{s}-\frac{1}{p}\right)+\left(\frac{1}{n}-\frac{1}{p}\right)$. Thus we may apply Hölder's inequality to the 
product

$$
\frac{|D u(y)|}{|x-y|^{n-1}}=\left(\frac{|D u(y)|^{n}}{|x-y|^{s(n-1)}}\right)^{1 / p} \cdot\left(\frac{1}{|x-y|^{s(n-1)}}\right)^{1 / s-1 / p} \cdot\left(|D u(y)|^{n}\right)^{1 / n-1 / p},
$$

obtaining

$$
\begin{aligned}
& \int_{B} \frac{|D u(y)|}{|x-y|^{n-1}} d y \leq\left(\int_{B} \frac{|D u(y)|^{n}}{|x-y|^{s(n-1)}} d y\right)^{1 / p} \\
& \quad \times\left(\int_{B} \frac{d y}{|x-y|^{s(n-1)}}\right)^{1 / s-1 / p} \cdot\left(\int_{B}|D u(y)|^{n} d y\right)^{1 / n-1 / p} .
\end{aligned}
$$

Set $a:=\int_{B}|D u(y)|^{n} d y$, since we have

$$
\begin{aligned}
\int_{B} & \frac{d y}{|x-y|^{s(n-1)}} \leq \int_{|z| \leq 2} \frac{d z}{|z|^{s(n-1)}} \\
& =\omega_{n-1} \int_{0}^{2} r^{((n-1)-s(n-1))} d r=\frac{\omega_{n-1} \cdot 2^{n-s(n-1)}}{n-s(n-1)} \\
& =\frac{\omega_{n-1} \cdot 2^{n-s(n-1)}}{n^{2}} \cdot((n-1) p+n) \leq \text { const } \cdot p,
\end{aligned}
$$

the inequalities (a.1) and (a.2) imply

$$
|u(x)|^{p} \leq \text { const } \cdot p^{p / s-1} \cdot a^{p / n-1} \cdot \int_{B} \frac{|D u(y)|^{n}}{|x-y|^{s(n-1)}} d y .
$$

Thus,

$$
\int_{B}|u(x)|^{p}|x|^{n \beta} d x \leq \mathrm{const} \cdot p^{p / s-1} \cdot a^{p / n-1} \cdot \int_{B} \int_{B} \frac{|D u(y)|^{n}}{|x-y|^{s(n-1)}} d y d x,
$$

(we have used $|x|^{n \beta} \leq 1$ which holds since $x \in B$ and $\beta>0$ ). By Fubini and (a.3) we have

$$
\int_{B} \int_{B} \frac{|D u(y)|^{n}}{|x-y|^{s(n-1)}} d y d x \leq \text { const } \cdot a \cdot p
$$

Therefore

$$
\left(\int_{B}|u|^{p} \cdot|x|^{n \beta} d x\right)^{1 / p} \leq \text { const } \cdot p^{1 / s} \cdot a^{1 / n} .
$$

But $p^{1 / s}=p^{(n-1) / n} \cdot p^{1 / p} \leq\left(e^{1 / e}\right) p^{(n-1) / n}$. Thus there exists a constant $c$ such that

$$
\left(\int_{B}|u|^{p} \cdot|x|^{n \beta} d x\right)^{1 / p} \leq c \cdot p^{(n-1) / n} \cdot a^{1 / n} .
$$

Second case. $-1<\beta \leq 0$. Set $t:=\frac{p}{2}\left(1-\frac{1}{\beta}\right), q:=p(1-\beta) /(1+\beta)$ (so that $\left.\frac{1}{t}+\frac{1}{q}=\frac{1}{p}\right)$. Applying Hölder's inequality to the product $|u(x)| \cdot|x|^{n \beta / p}$, 
we obtain

$$
\left(\int_{B}|u|^{p} \cdot|x|^{n \beta} d x\right)^{1 / p} \leq\left(\int_{B}|u|^{q} d x\right)^{1 / q} \cdot\left(\int_{B}|x|^{n \beta t / p} d x\right)^{1 / t} .
$$

Since $\frac{n \beta t}{p}>-n$ (for $\beta>-1$ ), we have $\int_{B}|x|^{n \beta t / p} d x=K<\infty$.

On the other hand, applying the proposition in the case $\beta=0$, we get

$$
\begin{aligned}
\left(\int_{B}|u|^{q} d x\right)^{1 / q} & \leq \text { const } \cdot q^{(n-1) / n}\left(\int_{B}|D u|^{n} d x\right)^{1 / n} \\
& \leq \text { const }\left(\frac{1-\beta}{1+\beta}\right)^{(n-1) / n} \cdot p^{(n-1) / n}\left(\int_{B}|D u|^{n} d x\right)^{1 / n} .
\end{aligned}
$$

Setting $C(B):=$ const $\cdot K \cdot\left(\frac{1-\beta}{1+\beta}\right)^{(n-1) / n}$, we have from the inequalities (a.8) and (a.9):

$$
\left(\int_{B}|u|^{p} \cdot|x|^{n \beta} \cdot d x\right)^{1 / p} \leq C(B) \cdot p^{(n-1) / n} \cdot\left(\int_{B}|D u|^{n} \cdot|x|^{\beta(n-p)} \cdot d x\right)^{1 / n}
$$

\section{REFERENCES}

1. T. Aubin, Nonlinear analysis on manifolds. Monge-Ampere equations, Springer-Verlag, New York, 1982.

2. M. S. Berger, Riemannian structure of prescribed Gaussian curvature for compact 2-manifolds, J. Differential Geom. 5 (1971), 325-332.

3. J. P. Bourguignon and J. P. Ezin, Scalar curvature functions in a conformal class of metrics and conformal transformations, Trans. Amer. Math. Soc. 301 (1987), 723-736.

4. P. Cherrier, Une inégalité de Sobolev sur les variétés riemanniennes, Bull. Sci. Math. 103 (1979), 353-374.

5. __ Problème de Neuman non linéaire sur les variétés riemanniennes, J. Funct. Anal. 57 (1984), 154-206.

6. S. A. Chang and P. Yang, Conformal deformation of metrics on $S^{2}$, J. Differential Geom. 27 (1988), 259-296.

7. R. Dautray and J. Lions, Analyse mathématique et calcul numérique pour les sciences et les techniques, Chapitre II, §3.2, Masson, Paris, 1987, pp. 335-349.

8. D. Gilbarg and N. S. Trudinger, Elliptic partial differential equation of second order, Springer Verlag, New York, 1977.

9. J. Kazdan, Prescribing the curvature of a Riemannian manifold, CBMS Regional Conf. Ser. in Math., no. 57, Amer. Math. Soc., Providence, R.I., 1985; reprinted 1988.

10. J. Kazdan and F. Warner, Curvature functions for compact 2-manifolds, Ann. of Math. 99 (1974), 14-47.

11. __ Existence and conformal deformation of metrics with prescribed Gaussian and scalar curvatures, Ann. of Math. 101 (1975), 317-331.

12. J. Moser, A sharp form of an inequality by Neil Trudinger, Indiana Univ. Math. J. 20 (1971), 1077-1092.

13. __ On a nonlinear problem in differential geometry, Dynamical System, edited by M. Peixoto, Academic Press, New York, 1973.

14. R. McOwen, Conformal metrics in $\mathbf{R}^{2}$ with prescribed Gaussian curvature and positive total curvature, Indiana Univ. Math. J. 34 (1985), 97-104.

15. Point singularities and conformal metrics on Riemann surfaces, Proc. Amer. Math. Soc. 103 (1988), 222-224. 
16. E. Picard, De l'intégration de l'équation $\Delta u=e^{u}$ sur une surface de Riemann fermée, Crelle's J. 130 (1905), 243-258.

17. M. Troyanov, Surfaces euclidiennes à singularités coniques, Enseign. Math. 32 (1986), 7994.

18. _ Les surfaces riemanniennes à singularités coniques, Thèse, Université de Genève, 1987.

19. __ Metrics of constant curvature on a sphere with two conical singularities, Proceedings of the Third International Symposium on Differential Geometry (Peniscola, 1988), Lecture Notes in Math., vol. 1410, Springer-Verlag, 296-308.

Centre de Mathematiques, École Polytechnique, F-91128 Palaiseau Cedex, France

Current address: Department of Mathematics, University of Utah, Salt Lake City, Utah 86112 